\title{
A Stick-Slip/Rouse Hybrid Model for Viscoelasticity in Polymers
}

\author{
H.T. Banks, J.B. Hood, N.G. Medhin and J.R. Samuels, Jr. \\ Center for Research in Scientific Computation \\ North Carolina State University \\ Raleigh, NC 27695-8205
}

November 19, 2006

\begin{abstract}
A Rouse model for polymer chains is incorporated into the linear continuous stickslip molecular-based tube reptation ideas of Doi-Edwards and Johnson-Stacer. This treats the physically constrained (PC) molecular stretches as internal strain variables for the overall PC/chemically cross-linked (CC) system. It yields an explicit system of stress-strain equations for the system permitting simple calculations of complex stressstrain relations. The model that is developed here treats PC molecule as entrapped within a constraining tube, which is comprised of both CC and PC molecules. The model is compared with experimental data sets from the literature.
\end{abstract}

\section{Introduction}

One of the most widely used empirical models for viscoelasticity in materials is the Boltzmann convolution law [15, 19, 20, 39]; for a nice summary and further references see Chapter 2 of [20]. In recent literature [5, 8, 9], models for hysteretic damping in elastomers entail a phenomenological Boltzmann-type constitutive law of the form

$$
\sigma(t)=g_{e}(\epsilon(t))+C_{D} \dot{\epsilon}(t)+\int_{-\infty}^{t} Y(t-s) \frac{d}{d s} g_{v}(\epsilon(s), \dot{\epsilon}(s)) d s
$$

where $Y$ is the convolution memory kernel, and $g_{e}$ and $g_{v}$ are nonlinear functions accounting for the elastic and viscoelastic responses of the elastomers, respectively. Previous efforts summarized in [6] have shown, through comparison with experimental data, that the best fit to filled elastomer data occurs when $g_{e}$ and $g_{v}$ are cubic, along with $Y$ as a distribution of exponentials. Banks, et al., [7,2] subsequently developed nonlinear models based on stick-slip "molecular" ideas of Johnson and Stacer [26] and Doi and Edwards [10] which resulted in a 
form for $g_{e}, g_{v}$ and $Y$ that matched the empirical findings reported in $[8,9,6]$. These models allow for multiple relaxation times present in polymer strands of composite materials within a virtual compartmental model of entangled chemically cross-linked/physically constrained system of long chain "molecules". While accounting for multiple relaxation parameters, the models do not include physically or chemically based parameters in the polymer strands.

In the present paper a new model is developed which combines the virtual stick-slip continuum "molecular-based" ideas of Johnson and Stacer [26] with the Rouse molecular-bead ideas as described in Doi and Edwards [10]. This new model, in which polymer chains are treated as strings of interconnected beads, permits the incorporation of many important physical parameters (such as temperature, segment bond length, internal friction, and segment density) in the overall hysteretic constitutive relationship. Our goal here is to present development of this model based upon physical considerations at the molecular level; its form is similar to that developed in [6,7] and does have the general form (1) of Boltzmann type, even though the kernel is not of convolution type,.

\section{Description of the Rouse Model}

For our summary of the Rouse model for free polymer strands and subsequent stress calculations, we follow for the most part the development in Doi and Edwards [10], modifying somewhat the random noise assumptions and the particular series used in order to insure convergence. We first assume a material composed of free polymer strands with each strand consisting of a finite set of beads connected in a string with elastic springs. Let $\left(\vec{R}_{1}, \vec{R}_{2}, \ldots, \vec{R}_{N}\right)$ be the position vectors relative to a fixed coordinate system of the beads comprising an interconnected chain as depicted schematically in Figure 1. Moreover, let the equation of motion of the beads be described by the Langevin equation [10]

$$
\frac{\partial}{\partial t} \vec{R}_{n}(t)=\sum_{m} H_{n m}\left(-\frac{\partial U}{\partial \vec{R}_{m}}+\vec{f}_{m}(t)\right)+\frac{1}{2} k_{B} T \sum_{m} \frac{\partial}{\partial \vec{R}_{m}} H_{n m},
$$

where $\vec{f}_{m}(t)$ is a random force term, $k_{B}$ is Boltzmann's constant, and $T$ is the temperature. The mobility tensor and the interaction potential are chosen to be

$$
\begin{gathered}
H_{n m}=\frac{\delta_{n m}}{\zeta} I, \\
U=\frac{k}{2} \sum_{n=2}^{N}\left\|\left(\vec{R}_{n}-\vec{R}_{n-1}\right)\right\|^{2},
\end{gathered}
$$

respectively, with

$$
k=\frac{3 k_{B} T}{b^{2}}
$$

where $b$ is the segment bond length at equilibrium and $\zeta$ is the friction constant of the polymer sample. 


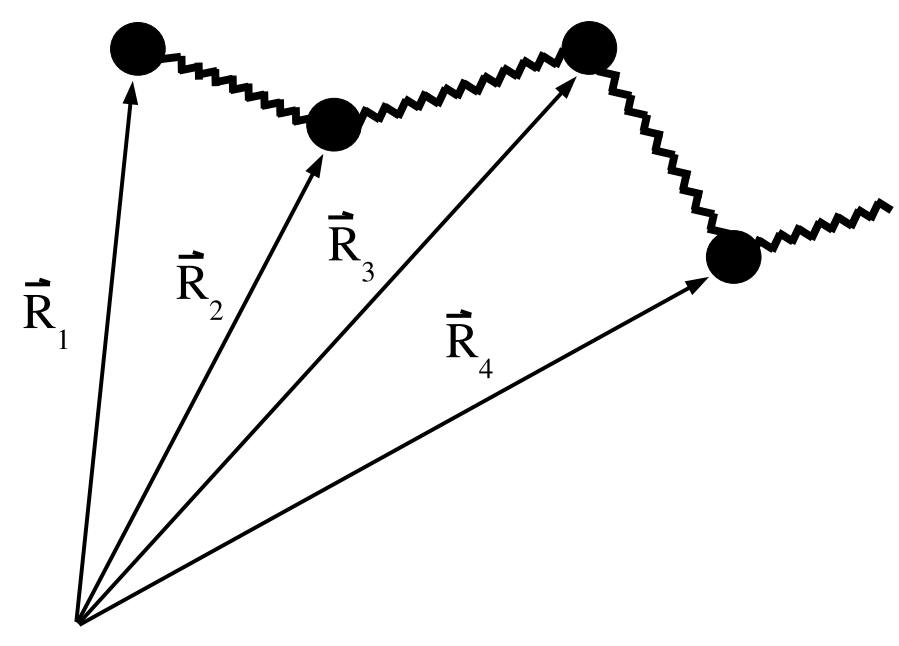

Figure 1: Representation of vectors for a bead-spring polymer molecule.

If we use the parameters defined above for the mobility tensor, $H_{n m}$, and for the interaction potential, $U$, then equation (2), for the cases when $n=2,3, \ldots, N-1$, can be written as

$$
\zeta \frac{d \vec{R}_{n}}{d t}=-k\left(2 \vec{R}_{n}-\vec{R}_{n+1}-\vec{R}_{n-1}\right)+\vec{f}_{n}
$$

For the special cases of the ends of the polymer, i.e., the cases when $n=1$ and $n=N$, we see that (respectively)

$$
\begin{gathered}
\zeta \frac{d \vec{R}_{1}}{d t}=-k\left(\vec{R}_{1}-\vec{R}_{2}\right)+\vec{f}_{1}, \\
\zeta \frac{d \vec{R}_{N}}{d t}=-k\left(\vec{R}_{N}-\vec{R}_{N-1}\right)+\vec{f}_{N}
\end{gathered}
$$

We define $\langle A\rangle$ to be the configurational average of the beads, i.e.,

$$
\langle A\rangle=\int A \psi(\vec{R} ; t) d \vec{R}
$$

where $\psi(\vec{R} ; t)$ is the configurational distribution [10] of the beads. The configurational distribution is a probability distribution which represents the probability that particles exist at the points $\left(\vec{R}_{1}, \ldots, \vec{R}_{N}\right)$ at the given time $t$.

The term $\overrightarrow{f_{n}}=\left(f_{n}^{1}, f_{n}^{2}, f_{n}^{3}\right)$ is a randomly distributed force which accounts for the Brownian motion of the beads. We assume therefore that the random force $\vec{f}_{n}$ is distributed 
according to a Gaussian distribution which is determined by the following moments

$$
\begin{gathered}
\left\langle\vec{f}_{n}(t)\right\rangle=0 \\
\left\langle f_{n}^{\alpha}(t) f_{m}^{\beta}\left(t^{\prime}\right)\right\rangle=2 \zeta k_{B} T \delta_{n m} \delta_{\alpha \beta} \delta\left(t-t^{\prime}\right),
\end{gathered}
$$

where $\delta_{i j}=1$ if $i=j$ and 0 otherwise, and $\delta(t)$ is the usual Dirac function.

To obtain the equation of a typical polymer strand from the finite bead strings, we conceptually take a continuum limit, replacing the system (4) with an equation on the interval $0 \leq n \leq N$, where now $N$ is the length of the strand. In the limit we obtain a partial differential equation in $n$ for the position $\vec{R}(t, n)$ of particles along the strand given by

$$
\begin{aligned}
& \zeta \frac{\partial \vec{R}(t, n)}{\partial t}=k \frac{\partial^{2} \vec{R}(t, n)}{\partial n^{2}}+\vec{f}(t, n), \text { for } 0 \leq n \leq N \\
& \left.\frac{\partial \vec{R}(t, n)}{\partial n}\right|_{n=0}=\left.\frac{\partial \vec{R}(t, n)}{\partial n}\right|_{n=N}=0 .
\end{aligned}
$$

This is obtained under the assumption that $\vec{R}_{0}=\vec{R}_{1}$ and $\vec{R}_{N+1}=\vec{R}_{N}$ and results from viewing the first term in the right side of (4) as a difference quotient for the second derivative. The generalized random force $\vec{f}(t, n)$ is now assumed to satisfy

$$
\begin{aligned}
& \langle\vec{f}(t, n)\rangle=0, \\
& \left\langle f^{\alpha}(t, n) f^{\beta}\left(t^{\prime}, m\right)\right\rangle=2 \zeta k_{B} T \delta(n-m) \delta_{\alpha \beta} \delta\left(t-t^{\prime}\right) .
\end{aligned}
$$

A standard method for analyzing systems such as (6)-(7) is via Fourier series with "modal" coordinates corresponding to the time dependent Fourier coefficients. A system of decoupled ordinary differential equations is obtained through separation of variables techniques. That is, we assume that $\vec{R}(t, n)$ can be expanded as

$$
\vec{R}(t, n)=\vec{X}_{0}(t)+\sqrt{\frac{2}{N}} \sum_{p=1}^{\infty} \vec{X}_{p}(t) \cos \left(\frac{p \pi n}{N}\right)
$$

in terms of the normalized Fourier elements $\varphi_{p}(n)=\sqrt{\frac{2}{N}} \cos \left(\frac{p \pi n}{N}\right)$. We further assume that the random noise has the form

$$
\begin{aligned}
\vec{f}(t, n) & =\sum_{p=1}^{\infty} \vec{g}_{p}(t) \sqrt{\frac{2}{N}} \cos \left(\frac{p \pi n}{N}\right) \\
& =\sum_{p=1}^{\infty} \mu_{p} \vec{W}_{p}(t) \sqrt{\frac{2}{N}} \cos \left(\frac{p \pi n}{N}\right)
\end{aligned}
$$

where the $\vec{W}_{p}(t)$ are Gaussian processes satisfying

$$
\left\langle\vec{W}_{p}(t)\right\rangle=0,
$$




$$
\left\langle W_{p}^{\alpha}(t) W_{q}^{\beta}\left(t^{\prime}\right)\right\rangle=\delta_{p q} \delta_{\alpha \beta} \delta\left(t-t^{\prime}\right) .
$$

The coefficients $\left\{\mu_{p}\right\}$ are chosen as

$$
\mu_{p}^{2}=\frac{12 \zeta k_{B} T N}{\pi^{2} p^{2}}, p=1,2, \ldots,
$$

so that all the infinite series in our subsequent discussions below converge and so that the relationship in (8) is satisfied. We then find that the modal coordinates $\vec{X}_{p}(t)$ are given by

$$
\vec{X}_{p}(t)=\sqrt{\frac{2}{N}} \int_{0}^{N} \cos \left(\frac{p \pi n}{N}\right) \vec{R}(t, n) d n, p=1,2, \ldots
$$

and satisfy

$$
\zeta \frac{\partial}{\partial t} \vec{X}_{p}=-k_{p} \vec{X}_{p}+\vec{g}_{p}
$$

where

$$
\begin{aligned}
& k_{p}=\frac{3 \pi^{2} k_{B} T}{N^{2} b^{2}} p^{2}, p=1,2, \ldots \\
& \left\langle g_{p}^{\alpha}(t)\right\rangle=0 \\
& \left\langle g_{p}^{\alpha}(t) g_{q}^{\beta}\left(t^{\prime}\right)\right\rangle=\delta_{p q} \delta_{\alpha \beta} \delta\left(t-t^{\prime}\right) \mu_{p}^{2} .
\end{aligned}
$$

\section{Stress Calculations}

Once the modal coordinates $\vec{X}_{p}$ have been found for the Rouse model for free polymer strands, it is possible to use them to determine a formula to approximate the stress tensor for a viscoelastic polymer undergoing deformations. We use the equation for the polymer dependent stress as given by equation (7.81) in [10], which is

$$
\sigma_{\alpha \beta}(t)=\frac{c}{N} \sum_{p=1}^{\infty} k_{p}\left\langle X_{p}^{\alpha}(t) X_{p}^{\beta}(t)\right\rangle
$$

where $c$ is the segment "density" (and thus $\frac{c}{N}$ represents the number per unit volume of polymer strands in the solid).

We define $\vec{R}(-0, n)$ to be the position of the polymer segment before deformation, and $\vec{R}(+0, n)$ to be the position of the segment immediately after deformation. Thus under the affine deformation assumption (e.g., see p. 241, [10]) which is a linearization approximation ( see p. 112, [10])

$$
\vec{R}(+0, n)=\mathbf{E}(0) \cdot \vec{R}(-0, n),
$$

or in terms of the modal coordinates,

$$
\vec{X}_{p}(+0)=\mathbf{E}(0) \cdot \vec{X}_{p}(-0),
$$


where the tensor $\mathbf{E}(0)=\left\{E_{\alpha \mu}(0)\right\}$ is the usual configuration gradient $\frac{\partial \vec{R}(+0)}{\partial \vec{R}(-0)}$ at time $t=0$. The matrix $\mathbf{E}$ is sometimes called the deformation gradient (in a misnomer) but the actual deformation gradient is $\mathbf{D}=\mathbf{E}-\mathbf{I}$ (see for example $[4,5,8,29,31]$ ). We recall that $\mathbf{E}$ can be used to define the Green-St. Venant strain $\mathcal{E}=\frac{1}{2}\left(\mathbf{E}^{T} \mathbf{E}-\mathbf{I}\right)=\frac{1}{2}\left(\mathbf{D}^{T} \mathbf{D}+\mathbf{D}+\mathbf{D}^{T}\right)$ as well as the left Cauchy-Green strain $\mathcal{E}^{L}=\mathbf{E E}^{T}$ which is the same as the Finger strain defined below. The equation (12) for $X_{p}^{\mu}(t)$ can be solved to obtain

$$
X_{p}^{\mu}(t)=X_{p}^{\mu}(0) e^{-\frac{p^{2}}{\tau_{R}} t}+\frac{1}{\zeta} \int_{0}^{t} g_{p}^{\mu}(s) e^{\frac{p^{2}}{\tau_{R}}(s-t)} d s,
$$

where

$$
\tau_{R}=\frac{\zeta N^{2} b^{2}}{3 \pi^{2} k_{B} T}
$$

is the Rouse relaxation time (p. 96, 196 of [10]). If we multiply $E_{\alpha \mu}$ on both sides of (17), we have

$$
E_{\alpha \mu} X_{p}^{\mu}(t)=E_{\alpha \mu} X_{p}^{\mu}(0) e^{-\frac{p^{2}}{\tau_{R}} t}+E_{\alpha \mu} \frac{1}{\zeta} \int_{0}^{t} g_{p}^{\mu}(s) e^{\frac{p^{2}}{\tau_{R}}(s-t)} d s
$$

In a similar manner we see that

$$
\left\langle E_{\alpha \mu} X_{p}^{\mu}(t) E_{\beta \nu} X_{p}^{\nu}(t)\right\rangle=E_{\alpha \mu} E_{\beta \nu}\left\langle X_{p}^{\mu}(t) X_{p}^{\nu}(t)\right\rangle .
$$

Noting from (9) that $\vec{g}_{p}(t)=\mu_{p} \vec{W}_{p}(t)$ and using (10), we find that the equation for the autocorrelation function is given by

$$
\left\langle X_{p}^{\alpha}(t) X_{p}^{\beta}(t)\right\rangle=\left\langle X_{p}^{\alpha}(0+) X_{p}^{\beta}(0+)\right\rangle e^{-2 \frac{p^{2}}{\tau_{R}} t}+\frac{\mu_{p}^{2}}{2 \zeta k_{p}} \delta_{\alpha \beta}\left(1-e^{-2 \frac{p^{2}}{\tau_{R}} t}\right) .
$$

We assume that the system is at equilibrium before the initial deformation at time $t=0$. We further assume that as $t$ approaches infinity, the system will return to its initial configuration (the equilibrium state). That is,

$$
\lim _{t \rightarrow \infty} X_{p}^{\alpha}(t)=X_{p}^{\alpha}(0-),
$$

which implies

$$
\left\langle X_{p}^{\mu}(0-) X_{p}^{\nu}(0-)\right\rangle=\frac{\mu_{p}^{2}}{2 \zeta k_{p}} \delta_{\mu \nu} .
$$

If the linearization approximation (16) is used, we find

$$
\left\langle X_{p}^{\alpha}(0+) X_{p}^{\beta}(0+)\right\rangle=B_{\alpha \beta}(\mathbf{E}(0)) \frac{\mu_{p}^{2}}{2 \zeta k_{p}},
$$

where

$$
B_{\alpha \beta}(\mathbf{E}(0))=\sum_{\mu=1}^{3} E_{\alpha \mu}(0) E_{\beta \mu}(0)=\left(\mathbf{E}(0) \mathbf{E}^{T}(0)\right)_{\alpha \beta}
$$


is the Finger strain (see p. 242, [10]). We note that the Finger strain is related to the Green-St. Venant strains through $B_{\alpha \beta}\left(\mathbf{E}^{T}\right)=2 \mathcal{E}_{\alpha \beta}+\delta_{\alpha \beta}$.

We may now substitute equation (20) into Equation (19), and we find that

$$
\left.\left\langle X_{p}^{\alpha}(t) X_{p}^{\beta}(t)\right\rangle=B_{\alpha \beta}(\mathbf{E}(0))\right) \frac{\mu_{p}^{2}}{2 \zeta k_{p}} e^{-2 \frac{p^{2}}{\tau_{R}} t}+\frac{\mu_{p}^{2}}{2 \zeta k_{p}} \delta_{\alpha \beta}\left(1-e^{-2 \frac{p^{2}}{\tau_{R}} t}\right) .
$$

From this expression we may note that without the $\mu_{p}$ terms as defined in (11), the resulting series for the stress tensor given by (14) does not converge! Also note equation (22) holds for $t>0$ small (see [10]).

\section{Connection with "Stick-Slip"}

Equation (22) coupled with (14) describes the contribution of each node of a free long chain polymer molecule to the molecule's overall stress. The goal of this work, however, is not to simply reproduce a stress-strain law based on a molecule in free space (i.e., based on the Langevin equation), but rather to describe the stress of a system composed of physically constrained (PC) molecules entangled with chemically cross-linked (CC) molecules and experiencing the stick-slip mechanisms. More precisely, we will view conceptually the material undergoing deformation as composed of two virtual compartments as depicted in Figure 2. One compartment will consist of a constraining tube which is a macroscopic compartment

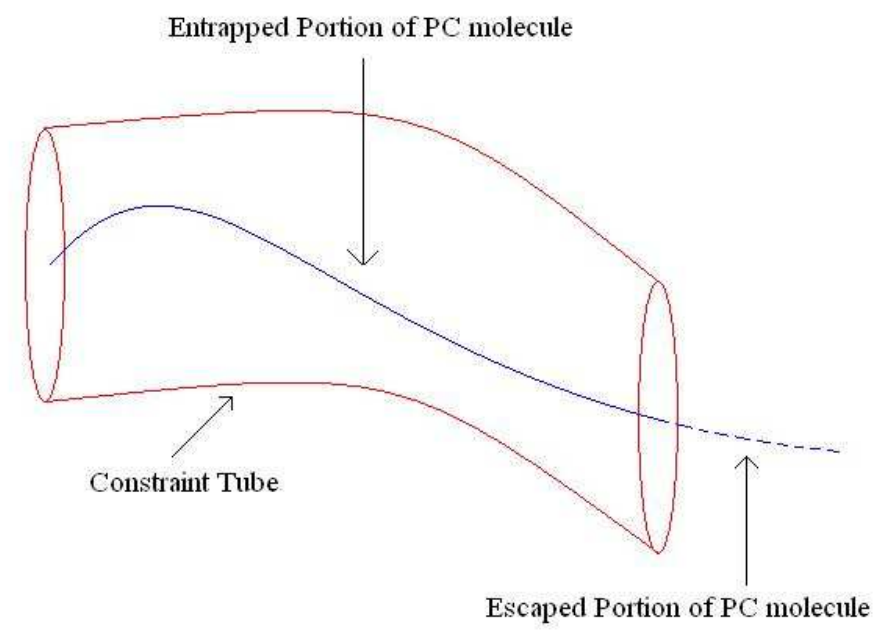

Figure 2: PC molecule entrapped by the surrounding constraining tube.

containing both $\mathrm{CC}$ and PC molecules. The other compartment will be microscopic in nature and consist of those PC molecules aligned with the direction of the deformation. These 
molecules will at first "stick" to the constraining tube and be carried along with its motion, but will very quickly "slip" and begin to "relax" back to a configuration of lower strain energy. We wish to compute the contributions of both "compartments" to the overall stress of a polymer material undergoing deformations.

To accomplish this goal we must consider the contribution from the constraining tube composed of both non-aligned physically constrained molecules and chemically cross-linked molecules, and that of PC molecules aligned in the direction of the deformation that are initially entangled with molecules of the tube. These aligned molecules will in time escape entanglement and become "free" molecules and will thus contribute to the overall stress in two distinct phases: when entrapped and after "leaking" free. Therefore, there are three contributions to the stress of the system $\sigma_{\alpha \beta}^{(P)}(t)$ : the PC chain in entanglement, the portion of the $\mathrm{PC}$ chain that has escaped entanglement, and the contribution due to the constraining tube. The constraining tube will be treated as elastic while we use the Rouse formulation to treat the aligned PC molecules. We will denote the stress of the portion of the polymer chain that is constrained by the surrounding molecules as $\sigma_{\alpha \beta}^{(1)}(t)$, and the stress of the portion of the polymer chain that has leaked out of the constraint tube as $\sigma_{\alpha \beta}^{(2)}(t)$. The total stress contribution of the entangled PC molecules will be denoted as

$$
\sigma_{\alpha \beta}^{(v e)}(t)=\sigma_{\alpha \beta}^{(1)}(t)+\sigma_{\alpha \beta}^{(2)}(t) .
$$

We will denote the stress of the constraining tube, assumed to be elastic, by $\sigma_{\alpha \beta}^{(\text {elas })}$. Thus, the total polymer dependent stress will be formulated as

$$
\sigma_{\alpha \beta}^{(P)}(t)=\sigma_{\alpha \beta}^{(v e)}(t)+\sigma_{\alpha \beta}^{(\text {elas })}(t) .
$$

We will use the Rouse model (14) in conjunction with a step-strain process (similar to the stick-slip molecular formulation of Johnson and Stacer [26]) to arrive at an appropriate form for $\sigma_{\alpha \beta}^{(v e)}(t)$. This will result in a hysteretic term as in a Boltzmann-type stress-strain law. To calculate the contribution of the entangled portion of the molecule to the stress we will subject the molecule to a series of instantaneous step-strains at times $0=t_{0}, t_{1}, \ldots, t_{n}$ with $\Delta t=t_{i}-t_{i-1}$ very small and investigate the behavior of the component $\left\langle X_{p}^{\alpha}(t) X_{p}^{\beta}(t)>\right.$ after each step-strain, where the PC molecules remaining in the tube are momentarily free and thus subject to the Rouse dynamics. We make, of course, the additional assumption that during the successive deformations some of the entrapped molecules will "leak" and escape entrapment (this "leaked" portion of the molecules will then be considered free, so we will therefore also assume the Rouse-like expression (22) to describe their motion). To treat the entrapped molecules we will let the time between each succeeding step-strain go to zero in order to obtain a constitutive law that describes each node's contribution to the stress after an instantaneous step-strain is applied to the molecules. To arrive at that point, 
first note in (22) that

$$
\begin{aligned}
\frac{\Delta\left\langle X_{p}^{\alpha}(0) X_{p}^{\beta}(0)\right\rangle}{\left\langle X_{p}^{\alpha}\left(0^{+}\right) X_{p}^{\beta}\left(0^{+}\right)\right\rangle} & =\frac{\left\langle X_{p}^{\alpha}\left(0^{+}\right) X_{p}^{\beta}\left(0^{+}\right)\right\rangle-\left\langle X_{p}^{\alpha}\left(0^{-}\right) X_{p}^{\beta}\left(0^{-}\right)\right\rangle}{\left\langle X_{p}^{\alpha}\left(0^{+}\right) X_{p}^{\beta}\left(0^{+}\right)\right\rangle} \\
& =\frac{B_{\alpha \beta}(\mathbf{E}(0)) \frac{\mu_{p}^{2}}{2 \zeta k_{p}}-\frac{\mu_{p}^{2}}{2 \zeta k_{p}} \delta_{\alpha \beta}}{B_{\alpha \beta}(\mathbf{E}(0)) \frac{\mu_{p}^{2}}{2 \zeta k_{p}}} \\
& =\frac{B_{\alpha \beta}(\mathbf{E}(0))-\delta_{\alpha \beta}}{B_{\alpha \beta}(\mathbf{E}(0))} \\
& =\frac{\Delta\left(B_{\alpha \beta}(\mathbf{E}(0))\right)}{B_{\alpha \beta}(\mathbf{E}(0))}
\end{aligned}
$$

where $\Delta B_{\alpha \beta}(\mathbf{E}(0)) \equiv B_{\alpha \beta}(\mathbf{E}(0))-\delta_{\alpha \beta}$ with $\delta_{\alpha \beta}$ representing the Finger strain in the undeformed state. A simple manipulation yields

$$
\left\langle X_{p}^{\alpha}\left(0^{+}\right) X_{p}^{\beta}\left(0^{+}\right)\right\rangle=\left\langle X_{p}^{\alpha}\left(0^{-}\right) X_{p}^{\beta}\left(0^{-}\right)\right\rangle+\frac{\left\langle X_{p}^{\alpha}\left(0^{+}\right) X_{p}^{\beta}\left(0^{+}\right)\right\rangle}{\left(B_{\alpha \beta}(\mathbf{E}(0))\right)} \Delta\left(B_{\alpha \beta}(\mathbf{E}(0))\right) .
$$

Since the PC molecule behaves according to Rouse's model momentarily after an instantaneous deformation (when it is still considered free), (19) implies

$$
\frac{\partial}{\partial t}\left\langle X_{p}^{\alpha}(t) X_{p}^{\beta}(t)\right\rangle=\frac{-2 p^{2}}{\tau_{R}}\left(\left\langle X_{p}^{\alpha}(t) X_{p}^{\beta}(t)\right\rangle-\frac{\mu_{p}^{2}}{2 \zeta k_{p}} \delta_{\alpha \beta}\right),
$$

or, equivalently,

$$
\frac{\partial}{\partial t}\left(\left\langle X_{p}^{\alpha}(t) X_{p}^{\beta}(t)\right\rangle-\frac{\mu_{p}^{2}}{2 \zeta k_{p}} \delta_{\alpha \beta}\right)=\frac{-2 p^{2}}{\tau_{R}}\left(\left\langle X_{p}^{\alpha}(t) X_{p}^{\beta}(t)\right\rangle-\frac{\mu_{p}^{2}}{2 \zeta k_{p}} \delta_{\alpha \beta}\right)
$$

and we determine on a short time interval $0=t_{0} \leq t \leq t_{1}$

$$
\begin{aligned}
\left\langle X_{p}^{\alpha}(t) X_{p}^{\beta}(t)\right\rangle & =\frac{\mu_{p}^{2}}{2 \zeta k_{p}} \delta_{\alpha \beta}+C e^{\frac{-2 p^{2}}{\tau_{R}} t} \\
& =\left\langle X_{p}^{\alpha}\left(0^{-}\right) X_{p}^{\beta}\left(0^{-}\right)\right\rangle+C e^{\frac{-2 p^{2}}{\tau_{R}} t} .
\end{aligned}
$$

According to (25) just after the step strain at $t=0^{+}$

$$
\begin{aligned}
\left\langle X_{p}^{\alpha}\left(0^{+}\right) X_{p}^{\beta}\left(0^{+}\right)\right\rangle & =\left\langle X_{p}^{\alpha}\left(0^{-}\right) X_{p}^{\beta}\left(0^{-}\right)\right\rangle+C e^{\frac{-2 p^{2}}{\tau_{R}} 0^{+}} \\
& =\left\langle X_{p}^{\alpha}\left(0^{-}\right) X_{p}^{\beta}\left(0^{-}\right)\right\rangle+C
\end{aligned}
$$

which, according to (24) implies

$$
C=\frac{\left\langle X_{p}^{\alpha}\left(0^{+}\right) X_{p}^{\beta}\left(0^{+}\right)\right\rangle}{\left(B_{\alpha \beta}(\mathbf{E}(0))\right)} \Delta\left(B_{\alpha \beta}(\mathbf{E}(0))\right)
$$


and

$$
\begin{aligned}
\left\langle X_{p}^{\alpha}(t) X_{p}^{\beta}(t)\right\rangle=\langle & \left.X_{p}^{\alpha}\left(0^{-}\right) X_{p}^{\beta}\left(0^{-}\right)\right\rangle \\
& +\frac{\left\langle X_{p}^{\alpha}\left(0^{+}\right) X_{p}^{\beta}\left(0^{+}\right)\right\rangle}{\left(B_{\alpha \beta}(\mathbf{E}(0))\right)} \Delta\left(B_{\alpha \beta}(\mathbf{E}(0))\right) e^{\frac{-2 p^{2}}{\tau_{R}}(t-0)}
\end{aligned}
$$

for $0=t_{0} \leq t \leq t_{1}$. The above procedure will serve as a basis for imitating Johnson and Stacer's step-strain procedure. In order to do so, we now need to make an assumption similar to $(24)$ at $t_{1}$. That is, we would expect

$$
\begin{aligned}
\frac{\Delta\left\langle X_{p}^{\alpha}\left(t_{1}\right) X_{p}^{\beta}\left(t_{1}\right)\right\rangle}{\left\langle X_{p}^{\alpha}\left(t_{1}^{+}\right) X_{p}^{\beta}\left(t_{1}^{+}\right)\right\rangle} & =\frac{\left\langle X_{p}^{\alpha}\left(t_{1}^{+}\right) X_{p}^{\beta}\left(t_{1}^{+}\right)\right\rangle-\left\langle X_{p}^{\alpha}\left(t_{1}^{-}\right) X_{p}^{\beta}\left(t_{1}^{-}\right)\right\rangle}{\left\langle X_{p}^{\alpha}\left(t_{1}^{+}\right) X_{p}^{\beta}\left(t_{1}^{+}\right)\right\rangle} \\
& \approx \frac{\Delta B_{\alpha \beta}\left(\mathbf{E}\left(t_{1}\right)\right)}{B_{\alpha \beta}\left(\mathbf{E}\left(t_{1}\right)\right)}
\end{aligned}
$$

or

$$
\begin{aligned}
\left\langle X_{p}^{\alpha}\left(t_{1}^{+}\right) X_{p}^{\beta}\left(t_{1}^{+}\right)\right\rangle=\langle & \left.X_{p}^{\alpha}\left(t_{1}^{-}\right) X_{p}^{\beta}\left(t_{1}^{-}\right)\right\rangle \\
& +\frac{\Delta B_{\alpha \beta}\left(\mathbf{E}\left(t_{1}\right)\right)}{B_{\alpha \beta}\left(\mathbf{E}\left(t_{1}\right)\right)}\left\langle X_{p}^{\alpha}\left(t_{1}^{+}\right) X_{p}^{\beta}\left(t_{1}^{+}\right)\right\rangle,
\end{aligned}
$$

where

$$
\Delta B_{\alpha \beta}\left(\mathbf{E}\left(t_{1}\right)\right)=B_{\alpha \beta}\left(\mathbf{E}\left(t_{1}+\Delta t\right)\right)-B_{\alpha \beta}\left(\mathbf{E}\left(t_{1}^{-}\right)\right) .
$$

Thus, it is necessary to understand what the quantity

$$
\left\langle X_{p}^{\alpha}\left(t_{i}^{+}\right) X_{p}^{\beta}\left(t_{i}^{+}\right)\right\rangle-\left\langle X_{p}^{\alpha}\left(t_{i}^{-}\right) X_{p}^{\beta}\left(t_{i}^{-}\right)\right\rangle
$$

in general represents. To investigate this quantity we compute

$$
\left\langle X_{p}^{\alpha}\left(t_{i}+\Delta t\right) X_{p}^{\beta}\left(t_{i}+\Delta t\right)\right\rangle-\left\langle X_{p}^{\alpha}\left(t_{i}^{-}\right) X_{p}^{\beta}\left(t_{i}^{-}\right)\right\rangle
$$

for $\Delta t>0$ and then let $\Delta t$ tend to zero from above. This procedure leads to the conclusion

$$
\frac{\left\langle X_{p}^{\alpha}\left(t_{i}+\Delta t\right) X_{p}^{\beta}\left(t_{i}+\Delta t\right)\right\rangle-\left\langle X_{p}^{\alpha}\left(t_{i}^{-}\right) X_{p}^{\beta}\left(t_{i}^{-}\right)\right\rangle}{\left\langle X_{p}^{\alpha}\left(t_{i}^{+}\right) X_{p}^{\beta}\left(t_{i}^{+}\right)\right\rangle} \approx \frac{\left[\Delta B_{\alpha \beta}\left(\mathbf{E}\left(t_{i}\right)\right) / \Delta t\right] \Delta t}{B_{\alpha \beta}\left(\mathbf{E}\left(t_{i}^{+}\right)\right)}
$$

where

$$
\Delta B_{\alpha \beta}\left(\mathbf{E}\left(t_{i}\right)\right)=B_{\alpha \beta}\left(\mathbf{E}\left(t_{i}+\Delta t\right)\right)-B_{\alpha \beta}\left(\mathbf{E}\left(t_{i}\right)\right) .
$$

We remark that

$$
\left.\lim _{\Delta t \rightarrow 0^{+}} \frac{\Delta B_{\alpha \beta}\left(\mathbf{E}\left(t_{i}\right)\right)}{\Delta t} \approx \frac{d}{d t} B_{\alpha \beta}(\mathbf{E}(t))\right|_{t=t_{i}}
$$


may either exist in the ordinary sense or may provide a jump at $t=t_{i}$. Arguments for this approximation and further details on the quantity

$$
\left\langle X_{p}^{\alpha}\left(t_{i}+\Delta t\right) X_{p}^{\beta}\left(t_{i}+\Delta t\right)\right\rangle-\left\langle X_{p}^{\alpha}\left(t_{i}^{-}\right) X_{p}^{\beta}\left(t_{i}^{-}\right)\right\rangle
$$

are found in the appendix.

Continuing with our arguments based on the Johnson and Stacer step-strain procedure, we recall from (29) and (30) that

$$
\begin{aligned}
\left\langle X_{p}^{\alpha}\left(t_{1}^{+}\right) X_{p}^{\beta}\left(t_{1}^{+}\right)\right\rangle= & \left\langle X_{p}^{\alpha}\left(0^{-}\right) X_{p}^{\beta}\left(0^{-}\right)\right\rangle+ \\
& \sum_{i=0}^{1} \frac{\left\langle X_{p}^{\alpha}\left(t_{i}^{+}\right) X_{p}^{\beta}\left(t_{i}^{+}\right)\right\rangle}{\left(B_{\alpha \beta}\left(\mathbf{E}\left(t_{i}\right)\right)\right)} \Delta\left(B_{\alpha \beta}\left(\mathbf{E}\left(t_{i}\right)\right)\right) e^{\frac{-2 p^{2}}{\tau_{R}}\left(t_{1}^{-}-t_{i}\right)} .
\end{aligned}
$$

Since Rouse's model requires $\left\langle X_{p}^{\alpha}(t) X_{p}^{\beta}(t)\right\rangle \stackrel{t \rightarrow \infty}{\longrightarrow}\left\langle X_{p}^{\alpha}(0-) X_{p}^{\beta}(0-)\right\rangle$ exponentially, we find

$$
\begin{aligned}
\left\langle X_{p}^{\alpha}(t) X_{p}^{\beta}(t)\right\rangle= & \left\langle X_{p}^{\alpha}\left(0^{-}\right) X_{p}^{\beta}\left(0^{-}\right)\right\rangle+\left(\sum_{i=0}^{1} \frac{\left\langle X_{p}^{\alpha}\left(t_{i}^{+}\right) X_{p}^{\beta}\left(t_{i}^{+}\right)\right\rangle}{\left(B_{\alpha \beta}\left(\mathbf{E}\left(t_{i}\right)\right)\right)} .\right. \\
& \left.\Delta\left(B_{\alpha \beta}\left(\mathbf{E}\left(t_{i}\right)\right)\right) e^{\frac{-2 p^{2}}{\tau_{R}}\left(t_{1}-t_{i}\right)}\right) e^{\frac{-2 p^{2}}{\tau_{R}}\left(t-t_{1}\right)}
\end{aligned}
$$

for $t_{1} \leq t \leq t_{2}$. If this process is repeated indefinitely, we find that

$$
\begin{aligned}
\left\langle X_{p}^{\alpha}(t) X_{p}^{\beta}(t)\right\rangle= & \left\langle X_{p}^{\alpha}\left(0^{-}\right) X_{p}^{\beta}\left(0^{-}\right)\right\rangle \\
& +\sum_{i=0}^{k} \frac{\left\langle X_{p}^{\alpha}\left(t_{i}^{+}\right) X_{p}^{\beta}\left(t_{i}^{+}\right)\right\rangle}{\left(B_{\alpha \beta}\left(\mathbf{E}\left(t_{i}\right)\right)\right)} \Delta\left(B_{\alpha \beta}\left(\mathbf{E}\left(t_{i}\right)\right)\right) e^{\frac{-2 p^{2}}{\tau_{R}}\left(t-t_{i}\right)}
\end{aligned}
$$

for $t>t_{k}$.

As mentioned above, we assume that some portion of the entrapped molecule can escape and behave as a free molecule. To account for these dynamics, we define $\gamma(t)$ to be the fraction of the molecule that is still entrapped at time $t$ (so that $\gamma(0)=1$ ). Recalling that $N$ is the length of the molecule, we define $N_{e}(t)=\gamma(t) N$ as the length of the molecule still entrapped at time $t$. Thus for the entrapped portion we have that

$$
\frac{\mu_{p}^{2}}{2 \zeta k_{p}}=\frac{2 b^{2} N_{e}^{3}}{\pi^{4} p^{4}}=\frac{2 b^{2} N^{3}}{\pi^{4} p^{4}} \gamma^{3}(t) .
$$

Returning to (22) we find if we let $N=N_{e}$ then the entrapped molecule contributes

$$
\begin{aligned}
\left\langle X_{p}^{\alpha}(t) X_{p}^{\beta}(t)\right\rangle= & \frac{2 b^{2} N^{3}}{\pi^{4} p^{4}} \gamma^{3}(t) B_{\alpha \beta}\left(\mathbf{E}\left(t_{0}\right)\right) e^{\frac{-2 p^{2}}{\tau_{R}}\left(t-t_{0}\right)} \\
& +\delta_{\alpha \beta} \frac{2 b^{2} N^{3}}{\pi^{4} p^{4}} \gamma^{3}(t)\left(1-e^{\frac{-2 p^{2}}{\tau_{R}}\left(t-t_{0}\right)}\right)
\end{aligned}
$$


to the stress for $0=t_{0} \leq t \leq t_{1}$, which leads to the approximation

$$
\frac{\left\langle X_{p}^{\alpha}\left(t_{0}^{+}\right) X_{p}^{\beta}\left(t_{0}^{+}\right)\right\rangle}{B_{\alpha \beta}\left(\mathbf{E}\left(t_{0}\right)\right)} \approx \frac{2 b^{2} N^{3}}{\pi^{4} p^{4}} \gamma^{3}\left(t_{0}\right) .
$$

Since the relaxation of the PC molecules obeys Rouses's model for a very short time after the instantaneous step-strain, it follows that on $t_{i} \leq t \leq t_{i+1}$, (32) holds with $t_{0}$ replaced by $t_{i}$. This leads immediately to

$$
\frac{\left\langle X_{p}^{\alpha}\left(t_{i}^{+}\right) X_{p}^{\beta}\left(t_{i}^{+}\right)\right\rangle}{B_{\alpha \beta}\left(\mathbf{E}\left(t_{i}\right)\right)} \approx \frac{2 b^{2} N^{3}}{\pi^{4} p^{4}} \gamma^{3}\left(t_{i}\right)
$$

for $t_{i} \leq t \leq t_{i+1}$. Doi and Edwards (p. 196, [10] or [21]) calculate

$$
\gamma(t)=\sum_{p \text { odd }} \frac{8}{\pi^{2} p^{2}} e^{\frac{-p^{2} t}{\tau_{d}}}
$$

where

$$
\tau_{d}=\frac{\zeta N^{3} b^{4}}{\pi^{2} k_{B} T a^{2}}
$$

is the disengagement time. If (34) is substituted into (31) we find

$$
\begin{aligned}
\left\langle X_{p}^{\alpha}(t) X_{p}^{\beta}(t)\right\rangle \approx & \left\langle X_{p}^{\alpha}\left(0^{-}\right) X_{p}^{\beta}\left(0^{-}\right)\right\rangle \\
& +\sum_{i=0}^{k} \frac{2 b^{2} N^{3}}{\pi^{4} p^{4}} \gamma^{3}\left(t_{i}\right) \frac{\Delta B_{\alpha \beta}\left(\mathbf{E}\left(t_{i}\right)\right)}{\Delta t} e^{\frac{-2 p^{2}}{\tau_{R}}\left(t-t_{i}\right)} \Delta t,
\end{aligned}
$$

for $t>t_{k}$. Taking the limit as $\Delta t=t-t_{i}$ goes to zero, we obtain

$$
\begin{aligned}
\left\langle X_{p}^{\alpha}(t) X_{p}^{\beta}(t)\right\rangle=\left\langle X_{p}^{\alpha}\left(0^{-}\right) X_{p}^{\beta}\left(0^{-}\right)\right\rangle & \\
& +\frac{2 b^{2} N^{3}}{\pi^{4} p^{4}} \int_{0}^{t} \gamma^{3}(s) \frac{d}{d s}\left(B_{\alpha \beta}(\mathbf{E}(s))\right) e^{\frac{-2 p^{2}}{\tau_{R}}(t-s)} d s .
\end{aligned}
$$

Therefore, the contribution to the stress of the constrained molecule is given by

$$
\begin{aligned}
\sigma_{\alpha \beta}^{(1)}(t)= & \frac{c}{N} \sum_{p=1}^{\infty} k_{p}\left\langle X_{p}^{\alpha}(t) X_{p}^{\beta}(t)\right\rangle \\
= & \frac{c}{N} \sum_{p=1}^{\infty} k_{p} \frac{\mu_{p}^{2}}{2 \zeta k_{p}} \delta_{\alpha \beta} \\
& +\frac{c}{N} \sum_{p=1}^{\infty} k_{p} \frac{2 b^{2} N^{3}}{\pi^{4} p^{4}} \int_{0}^{t} \gamma^{3}(s) \frac{d}{d s}\left(B_{\alpha \beta}(\mathbf{E}(s))\right) e^{\frac{-2 p^{2}}{\tau_{R}}(t-s)} d s \\
= & \sum_{p=1}^{\infty} \frac{6 c k_{B} T}{\pi^{2} p^{2}}\left(\delta_{\alpha \beta}+\int_{0}^{t} \gamma^{3}(s) \frac{d}{d s}\left(B_{\alpha \beta}(\mathbf{E}(s))\right) e^{\frac{-2 p^{2}}{\tau_{R}}(t-s)} d s\right) \\
= & \sum_{p=1}^{\infty} \frac{6 c k_{B} T}{\pi^{2} p^{2}} \delta_{\alpha \beta}+\int_{0}^{t} Y(t, s) \frac{d}{d s}\left(B_{\alpha \beta}(\mathbf{E}(s))\right) d s
\end{aligned}
$$


where $Y(t, s) \equiv \gamma^{3}(s) Y(t-s)$ with

$$
Y(t-s)=\frac{6 c k_{B} T}{\pi^{2}} \sum_{p=1}^{\infty} \frac{1}{p^{2}} e^{\frac{-2 p^{2}}{\tau_{R}}(t-s)}=\sum_{p=1}^{\infty} c_{p} e^{-\frac{1}{\tau_{p}}(t-s)}
$$

for

$$
c_{p}=\frac{6 c k_{B} T}{\pi^{2} p^{2}} \quad \text { and } \quad \tau_{p}=\frac{\tau_{R}}{2 p^{2}} .
$$

We note that this stress term can be written in terms of the left Cauchy-Green strain $\mathcal{E}^{L}$ as

$$
\sigma_{\alpha \beta}^{(1)}(t)=\sum_{p=1}^{\infty} \frac{6 c k_{B} T}{\pi^{2} p^{2}} \delta_{\alpha \beta}+\int_{0}^{t} Y(t, s) \frac{d}{d s}\left(\mathcal{E}_{\alpha \beta}^{L}(s)\right) d s .
$$

Observe that the form of $\sigma^{(1)}(t)$ is the similar to that of the Boltzmann-type stress-strain law (1) except that the kernel $Y$ is no longer in simple convolution form as in (35).

The contribution to the stress due to the portion of each polymer chain that has leaked out of the constraint tube is given by a modification of (22) similar to the one used in (32) above

$$
\begin{aligned}
\sigma_{\alpha \beta}^{(2)}(t)= & \frac{c}{N} \sum_{p=1}^{\infty} k_{p} \frac{\mu_{p}^{2}}{2 \zeta k_{p}} B_{\alpha \beta}(\mathbf{E}(0)) e^{\frac{-2 p^{2}}{\tau_{R}} t} \\
& +\frac{c}{N} \sum_{p=1}^{\infty} k_{p} \frac{\mu_{p}^{2}}{2 \zeta k_{p}} \delta_{\alpha \beta}\left(1-e^{\frac{-2 p^{2}}{\tau_{R}} t}\right) \\
= & \sum_{p=1}^{\infty} \frac{6 c k_{B} T}{\pi^{2} p^{2}}(1-\gamma(t))^{3} B_{\alpha \beta}(\mathbf{E}(0)) e^{\frac{-2 p^{2}}{\tau_{R}} t} \\
& +\sum_{p=1}^{\infty} \frac{6 c k_{B} T}{\pi^{2} p^{2}}(1-\gamma(t))^{3} \delta_{\alpha \beta}\left(1-e^{\frac{-2 p^{2}}{\tau_{R}} t}\right) .
\end{aligned}
$$

Finally, for the contribution $\sigma_{\alpha \beta}^{(\text {elas })}(t)$ of the elastic constraining tube to the overall stress in (23) we choose the Finger strain Hookean form

$$
\sigma_{\alpha \beta}^{(\text {elas })}(t)=\mu^{Y} B_{\alpha \beta}(\mathbf{E}(t))
$$

where $\mu^{Y}$ is a generalized Young's modulus of elasticity.

\section{Uniaxial Deformation}

In this section we consider uniaxial deformations and examine the equation for the macroscopic stress [10], which is of the form

$$
\sigma_{\alpha \beta}(t)=\sigma_{\alpha \beta}^{(P)}(t)+P \delta_{\alpha \beta} .
$$


Here the term $\sigma_{\alpha \beta}^{(P)}$ represents the contribution from the polymer molecules (the polymer dependent stress, as defined in equation (23)) and $P$ is the hydrostatic pressure.

We proceed by assuming that we are applying a tensile deformation, i.e., a deformation strictly in one of the three principle directions (specifically, we will consider a stretch in the $z=x_{3}$ direction). To determine the stress for such a deformation, first the appropriate Finger strain is required. If we consider a unit cube, and apply a small deformation in the $z$ direction, then it attains a length of

$$
\lambda=1+\epsilon,
$$

where the strain, $\epsilon$, is the ratio of the change in length, $\Delta L$, to the original length, $L$, or in other words, $\epsilon=\frac{\Delta L}{L}$. If the material is assumed incompressible, the volume must be maintained. Thus the sides in the $x=x_{1}$ and $y=x_{2}$ direction must both necessarily be of length $\frac{1}{\sqrt{\lambda}}$.

We choose a random point within the solid denoted by $\hat{R}=\left(\hat{R}^{1}, \hat{R}^{2}, \hat{R}^{3}\right)$. The point's change in position after deformation from $\hat{R}$ to the new location, denoted by $\tilde{R}$, can be described, to first order, by the equations (p. 241, [10])

$$
\tilde{R}^{1}=\frac{1}{\sqrt{\lambda}} \hat{R}^{1}, \quad \tilde{R}^{2}=\frac{1}{\sqrt{\lambda}} \hat{R}^{2}, \quad \tilde{R}^{3}=\lambda \hat{R}^{3} .
$$

These equations give a configuration gradient $\mathbf{E}$ of the form

$$
\mathbf{E}=\left[\begin{array}{ccc}
\frac{1}{\sqrt{\lambda}} & 0 & 0 \\
0 & \frac{1}{\sqrt{\lambda}} & 0 \\
0 & 0 & \lambda
\end{array}\right]
$$

which provides a Finger strain of the form

$$
B(\mathbf{E})=\left[\begin{array}{ccc}
\frac{1}{\lambda} & 0 & 0 \\
0 & \frac{1}{\lambda} & 0 \\
0 & 0 & \lambda^{2}
\end{array}\right] .
$$

We define the tensile stress $\Sigma_{\alpha}$ in the principle direction using the macroscopic stress given by equation (38)

$$
\Sigma_{\alpha}=\sigma_{\alpha \alpha}^{(P)}+P
$$

where $P$ is a finite hydrostatic pressure term $[11,31]$. For our case of deformation in the $z$ direction, we consider the equation

$$
\Sigma_{z}=\sigma_{z z}^{(P)}+P
$$

in which we must determine the hydrostatic pressure term $P$. This is done by noting that since the deformation is uniaxial in the $z$-direction, no force acts in the $x$ - or $y$-directions. 
Thus, the stress in the $x$ - and $y$-directions vanishes, i.e., $\Sigma_{x}=\Sigma_{y}=0$, and from the equation $\Sigma_{x}=\sigma_{x x}^{(P)}+P$ for the tensile stress in the $x$ direction, it is seen that $P=-\sigma_{x x}^{(P)}$.

Thus, if a tensile deformation is performed on the elastomer along the $z$ axis then the stress is given by

$$
\begin{aligned}
\Sigma_{z}(t)= & \sigma_{z z}^{(P)}(t)+P \\
= & \sigma_{z z}^{(P)}(t)-\sigma_{x x}^{(P)}(t) \\
= & \sigma_{z z}^{(v e)}(t)-\sigma_{x x}^{(v e)}(t)+\sigma_{z z}^{(\text {elas })}(t)-\sigma_{x x}^{(\text {elas })}(t) \\
= & \sigma_{z z}^{(1)}(t)-\sigma_{x x}^{(1)}(t)+\sigma_{z z}^{(2)}(t)-\sigma_{x x}^{(2)}(t)+\sigma_{z z}^{(\text {elas })}(t)-\sigma_{x x}^{(\text {elas })}(t) \\
= & \frac{6 c k_{B} T}{\pi^{2}} \sum_{p=1}^{\infty}\left(\frac{1}{p^{2}} \int_{0}^{t} \gamma^{3}(s) e^{-\frac{2 p^{2}}{\tau_{R}}(t-s)}\left[2 \lambda(s) \lambda^{\prime}(s)+\frac{\lambda^{\prime}(s)}{\lambda^{2}(s)}\right] d s\right. \\
& \left.\quad+\frac{(1-\gamma(t))^{3}}{p^{2}}\left(\lambda^{2}(0)-\frac{1}{\lambda(0)}\right) e^{\frac{-2 p^{2}}{\tau_{R}} t}\right)+\mu^{Y}\left(\lambda^{2}(t)-\frac{1}{\lambda(t)}\right) .
\end{aligned}
$$

The term $\mu^{Y}\left(\lambda^{2}-\frac{1}{\lambda}\right)$, where $\mu^{Y}$ is the Young's modulus of elasticity, accounts for the stress contribution of the elastic constraining tube. We observe that this corresponds to the Cauchy or true stress for an incompressible neo-Hookean material undergoing uniaxial elongation. This can be derived in a pseudo-phenomenological approach $[4,5,8]$ using the Mooney strain energy function (SEF) in the context of a nonlinear elasticity approach [29, 31, 34, 38, 39].

\section{Parameter Estimation and Simulation Results}

\subsection{Articular cartilage results}

In this subsection, we report on calculations performed with the stress-strain relationship (39) using parameters determined from a set of data from experiments on articular cartilage (a material of significant scientific interest which is widely viewed as a viscoelastic materialsee [21] and the references therein). This will provide a first test of our stress models in reproducing results from other models and physical experiments. First the appropriate parameters used in the model are estimated in inverse problems incorporating the data. Then stress calculations which are based on the results of experimental work will be presented. The stress-strain relation will be evaluated by calculating the stress and comparing it to experiments for various input strain functions. Finally, simulations are conducted to repeat the results of Johnson and Stacer's paper [26] on which the model is partially based.

\subsubsection{Estimating parameters and corresponding stress-strain simulations}

The experiments conducted by Huang, et.al., [24] involved applying a tensile strain (deformation) to a sample of articular cartilage and then measuring the stress within the cartilage. Two such experiments were conducted in which two different input strains were used. These 
strains were ramp strains starting at zero and increasing at a constant rate until a cessation time $\left(t_{s}\right)$. The material was then held at a fixed strain $\epsilon_{\max }$ until the experiment terminates at time $t_{f}=2000$ seconds. For both experiments $\epsilon_{\max }$ was taken to be 0.05 , while the first had a cessation time of $t_{s}^{1}=.126$ seconds and the second had a cessation time of $t_{s}^{2}=400$ seconds. Thus the equation for the strain functions is given by

$$
\epsilon_{i}(t)= \begin{cases}\frac{\epsilon_{\max }}{t_{s}^{i}} t & t \leq t_{s}^{i} \\ \epsilon_{\max } & t>t_{s}^{i}\end{cases}
$$

for $i=1,2$. The chosen parameters used in the strain function for these experiments are presented in Table 1 while the graphs of the strain functions are given in Figure 3.

Table 1: Parameters used for ramp input strain function for cartilage stress experiments.

\begin{tabular}{|l|c|l|}
\hline Parameter & Abbreviation & Value \\
\hline Cessation Time 1 & $t_{s}^{1}$ & .126 seconds \\
Cessation Time 2 & $t_{s}^{2}$ & 400 seconds \\
Maximum Strain & $\epsilon_{\max }$ & 0.05 \\
\hline
\end{tabular}

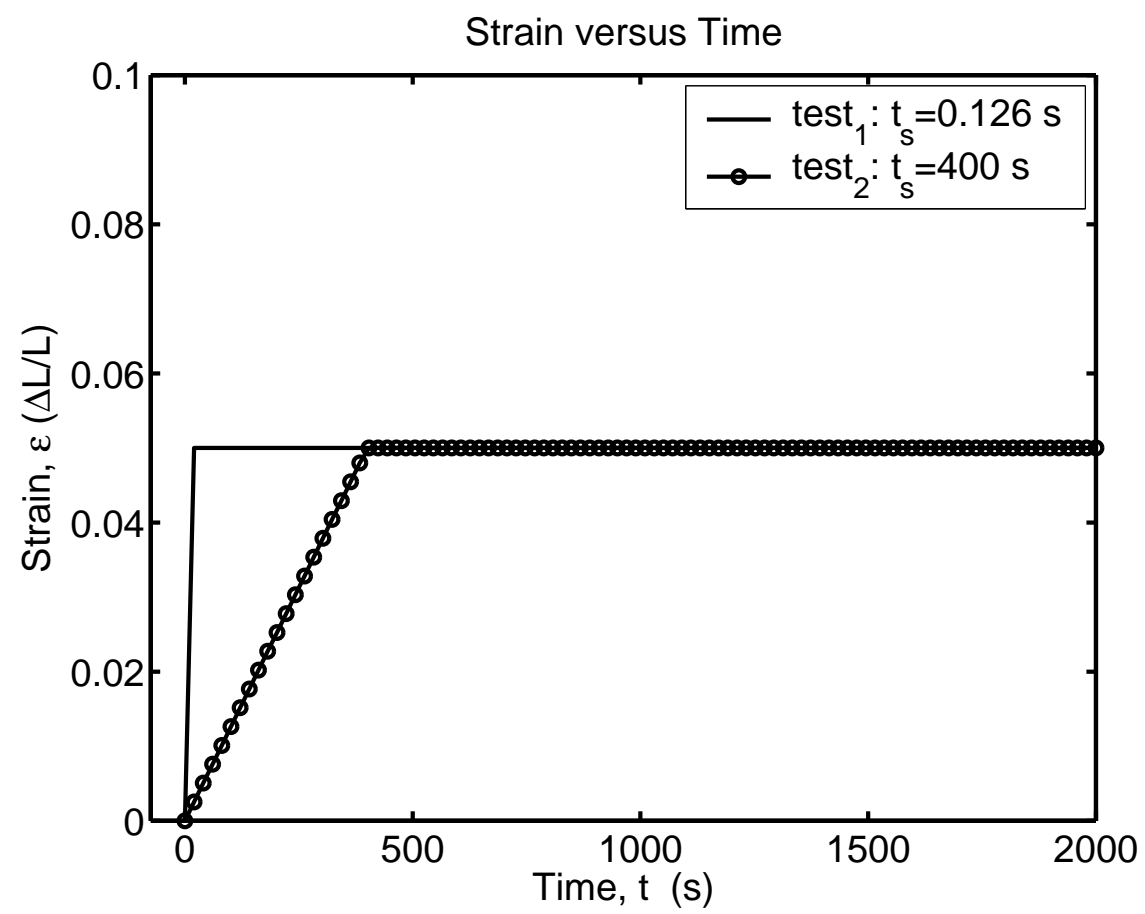

Figure 3: Input strains used for stress calculations in cartilage stress simulation.

It is assumed that the experiments were conducted at room temperature which is taken to be $T=300^{\circ} \mathrm{K}$. For the length of the polymer chain we chose $N=1000$. A sensitivity 
analysis of the model (39) with respect to parameters such as the step length $a$, bond length $b$, segment density $c$, the frictional constant $\zeta$ and the constraining tube's elastic constant $\mu=\mu^{Y}$ was carried out to determine possible correlations. Based on these investigations the model was re-parameterized by defining $\tilde{a}=\frac{b^{4} \zeta}{a^{2}}$ (the parameter characterizing $\left.\tau_{D}\right), \tilde{b}=b^{2} \zeta$ (the parameter characterizing $\tau_{R}$ ), and $\tilde{\mu}=\frac{\mu^{Y}}{c}$ (a normalized Young's constant). (We remark that in view of (43) and (44) below, we could have included $N^{2}$ and $N^{3}$ in the scaled variables $\tilde{b}$ and $\tilde{a}$, respectively, but the estimation results would have ultimately produced the same fits to data in the efforts with experimental data reported on below.) To obtain values for the parameters $\tilde{a}, \tilde{b}, c$, and $\tilde{\mu}$, parameter estimation methods using the experimental data with the model were employed .

To perform the parameter estimation, data was extracted from the graphs presented in Figure 7 of [24]. These graphs depict the stress on articular cartilage for applied ramp strains as described above. (Graphs of the extracted data are presented as solid lines in Figure 4.) The data was extracted using the MatLab tool Grabit, written by Jiro Doke [12]. Two sets of data were obtained, one from each of the experiments performed, and these are referred to as $\left\{y_{d}^{1 j}\right\}_{j=1}^{100}$ and $\left\{y_{d}^{2 j}\right\}_{j=1}^{100}$, where $y_{d}^{i j}$ is the stress value for the strain function $\epsilon_{i}\left(t_{j}\right)$ as described by equation (40)) at time $t_{j}$.

The data is then used in a weighted least-squares cost function to determine the optimal values for the desired parameters in a vector

$$
\vec{\theta}=\left[\begin{array}{c}
\theta_{1} \\
\theta_{2} \\
\theta_{3} \\
\theta_{4}
\end{array}\right]=\left[\begin{array}{c}
\tilde{a} \\
\tilde{b} \\
c \\
\tilde{\mu}
\end{array}\right] .
$$

The weighted least squares (WLS) function is given by

$$
C(\vec{\theta})=\sum_{i=1}^{2}\left(\frac{1}{\max \left\{y_{d}^{i}\right\}}\right)^{2}\left[\sum_{j=1}^{100}\left|\Sigma_{z}\left(t_{j} ; \vec{\theta}, i\right)-y_{d}^{i j}\right|^{2}\right],
$$

with which we employed a Nelder-Mead method (fminsearch in MatLab) to determine the optimal value of the parameter vector $\vec{\theta}$. In this case all of the parameters we seek are uniquely defined. The response function $\Sigma_{z}$ is defined by letting

$$
\Lambda_{i}(s)=2 \lambda_{i}(s) \lambda_{i}^{\prime}(s)+\frac{\lambda_{i}^{\prime}(s)}{\lambda_{i}^{2}(s)},
$$

and then defining the function

$$
\begin{aligned}
\Sigma_{z}\left(t_{j} ; \vec{\theta}, i\right)= & \theta_{3}\left[\frac { 6 k _ { B } T } { \pi ^ { 2 } } \sum _ { p = 1 } ^ { M } \frac { 1 } { p ^ { 2 } } \left\{\int_{0}^{t_{j}} \gamma\left(s ; \theta_{1}\right)^{3} \Lambda_{i}(s) e^{-\frac{6 p^{2} \pi^{2} k_{B} T}{\theta_{2} N^{2}}\left(t_{j}-s\right)} d s\right.\right. \\
& \left.+\left(1-\gamma\left(s ; \theta_{1}\right)\right)^{3}\left(\lambda_{i}^{2}(0)-\frac{1}{\lambda_{i}(0)}\right) e^{-\frac{6 p^{2} \pi^{2} k_{B} T}{\theta_{2} N^{2}} t_{j}}\right\} \\
& \left.+\theta_{4}\left(\lambda_{i}^{2}\left(t_{j}\right)-\frac{1}{\lambda_{i}\left(t_{j}\right)}\right)\right] .
\end{aligned}
$$


The summation limit $M$ was chosen to be 10 since it was found that the total sum would change by less than $1 \%$ if $M$ were increased. In computing the integrals in (43), an approximation was made using the MatLab quad routine, which utilizes a Simpson's quadrature method to approximate the integral. We also used

$$
\gamma\left(t ; \theta_{1}\right)=\sum_{p \text { odd }}^{M^{\prime}} \frac{8}{p^{2} \pi^{2}} \exp \left(\frac{-p^{2} \pi^{2} k_{B} T}{\theta_{1} N^{3}} t\right),
$$

where the limit of summation was chosen to be $M^{\prime}=21$ (for a larger $M^{\prime}$ value, the increase in the sum is not sufficient to justify the increased computation time). The term $\lambda_{i}(t)=1+\epsilon_{i}(t)$ is defined for $\epsilon_{i}(t)$, as given above. The derivative of $\lambda_{i}(t)$ was taken for $i=1,2$, as

$$
\dot{\epsilon}_{i}(t)=\left\{\begin{array}{ll}
\frac{\epsilon_{\max }}{t_{s}^{i}} & t \leq t_{s}^{i} \\
0 & t>t_{s}^{i}
\end{array} .\right.
$$

The cost function was calculated using equation (43) in the minimization algorithms. These calculations require an initial guess, denoted by $\overrightarrow{\theta_{0}}$, for the value of $\vec{\theta}$. Since little can be found in the literature for these parameters in the case of cartilage, in this section we obtained initial values $\overrightarrow{\theta_{0}}$ by simulating with the model with numerous parameter values over a wide range and comparing the corresponding graphs visibly with the data. A physically-based method for determining initial estimates in the case where one knows rough parameter ranges for a material is described for the case of polyisoprene data in the next section.

In addition to calculating the optimal values of the parameters in $\vec{\theta}$ we will determine the standard errors for $\tilde{a}, \tilde{b}$ and $\tilde{\mu}$. The process to calculate the standard errors depends upon the form of the cost functional. If the ordinary least squares (OLS) cost functional is used (as it will later in (45)) then the $k^{\text {th }}$ standard error is approximated as

$$
S E_{k}\left(\hat{\theta}^{n}\right)=\sqrt{\mathcal{C}_{k k}\left(\hat{\theta}^{n}\right)}
$$

The term $\mathcal{C}_{k k}$ is the $k^{t h}$ diagonal element of the $M \times M$ covariance matrix

$$
\mathcal{C}\left(\hat{\theta}^{n}\right)=\hat{\sigma}^{2}\left[\chi^{T}\left(\hat{\theta}^{n}\right) \chi\left(\hat{\theta}^{n}\right)\right]^{-1}
$$

where

$$
\chi_{j k}(\theta)=\frac{\partial f_{j}(\theta)}{\partial \theta_{k}}
$$

is the $(j, k)$ element of the sensitivity matrix $\chi \in \Re^{n \times M}, \hat{\theta}^{n} \in \Re^{M}$ is the parameter estimate obtained in the optimization process, and

$$
\hat{\sigma}^{2}=\frac{1}{n-M} \sum_{j=1}^{n}\left|f_{j}\left(\hat{\theta}^{n}\right)-y_{j}\right|^{2}
$$


with $f_{j}\left(\hat{\theta}^{n}\right)=\Sigma_{z}\left(t_{j} ; \hat{\theta}^{n}\right)$ denoting the model's value at time $t_{j}$ and parameter estimate $\hat{\theta}^{n}$. More details regarding large sample size approximation statistics can be found in the standard nonlinear regression approximation theory $([13,22,25]$, and Chapter 12 of [35]). For a brief summary also see Section 3 of [3].

If, on the other hand, a weighted least squares cost functional is used (as in (42)) a matrix that accounts for the individual weights must be included in the formation of the covariance matrix

$$
\mathcal{C}\left(\hat{\theta}^{n}\right)=\hat{\sigma}^{2}\left[\chi^{T}\left(\hat{\theta}^{n}\right) W\left(\hat{\theta}^{n}\right) \chi\left(\hat{\theta}^{n}\right)\right]^{-1} .
$$

The weights used in (42) produce the weighting matrix

$$
W^{-1}(\theta)=\operatorname{diag}\left(\max \left\{y_{d}^{1}\right\}, \ldots, \max \left\{y_{d}^{1}\right\}, \max \left\{y_{d}^{2}\right\}, \ldots, \max \left\{y_{d}^{2}\right\}\right)
$$

We note that the segment density $c$ acts as a scaling parameter for the model and hence requires a single data point to set its value. Therefore, analysis reveals that the model is relatively insensitive to changes in $c$, making a standard error calculation involving multiple observations irrelevant.

For the case when $\overrightarrow{\theta_{0}}$ is taken to be

$$
\overrightarrow{\theta_{0}}=\left[\begin{array}{c}
7 \times 10^{-6} \\
9 \times 10^{-4} \\
10^{-3} \\
10^{2}
\end{array}\right]
$$

the optimal value returned by the MatLab program is

$$
\vec{\theta}_{\text {opt }}=\left[\begin{array}{c}
3.4592 \times 10^{-5} \pm 1.1828 \times 10^{-25} \\
4.0786 \times 10^{-3} \pm 3.3634 \times 10^{-24} \\
5.7831 \times 10^{-4} \\
1.5587 \times 10^{2} \pm 1.1383 \times 10^{-23}
\end{array}\right] .
$$

These optimal values, along with the other important values associated with the calculation of the stress function, are presented in Table 2.

Table 2: Fixed and optimal parameter values used for cartilage stress simulations

\begin{tabular}{|l|c|l|}
\hline Parameter & Abbreviation & Value \\
\hline Temperature & $T$ & $300^{\circ} \mathrm{K}$ \\
Segments/Chain & $N$ & 1000 \\
Boltzmann's Constant & $k_{B}$ & $1.3806505 \times 10^{-23} \mathrm{~J} / \mathrm{K}$ \\
Disengagement constant & $\tilde{a}_{\text {opt }}$ & $3.4592 \times 10^{-5} \AA^{2} \mathrm{~kg} / \mathrm{s}$ \\
Rouse constant & $\tilde{b}_{\text {opt }}$ & $4.0786 \times 10^{-3} \AA^{2} \mathrm{~kg} / \mathrm{s}$ \\
Segments/Volume & $c_{\text {opt }}$ & $5.7831 \times 10^{-4} 1 / \AA^{3}$ \\
Young's constant & $\tilde{\mu}_{\text {opt }}$ & $1.5587 \times 10^{2} \mathrm{MPa} \AA^{3}$ \\
\hline
\end{tabular}


Once we have determined a set of parameters which provide an optimal fit to the data obtained from the paper by Huang, et al., we now can perform calculations which will simulate the experiments.

The resulting simulations are presented in Figure 4 in a comparison with the data obtained from [24].

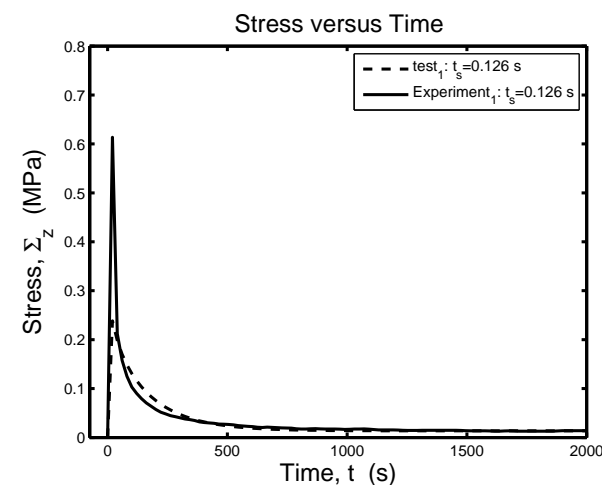

Cartilage Experiment 1

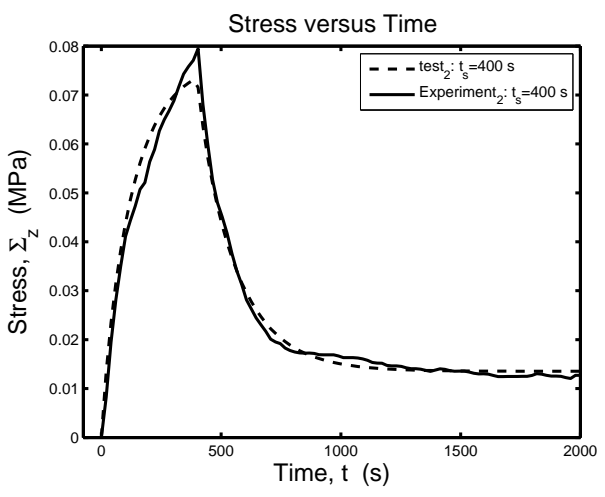

Cartilage Experiment 2

Figure 4: Stress calculation with optimal fit parameters versus experimental data for cartilage with ramp strain inputs.

Instead of using both sets of data to obtain $\vec{\theta}_{\text {opt }}$ as we did in minimizing (42), we could also obtain a set of optimal parameters for each experiment separately. That is, we could use

$$
C_{i}(\vec{\theta})=\sum_{j=1}^{100}\left|\Sigma_{z}\left(t_{j} ; \vec{\theta}, i\right)-y_{d}^{i j}\right|^{2},
$$

to obtain separate optimal parameters $\vec{\theta}_{\text {opt }}^{i}, i=1,2$, for each experiment. When the experiments are considered separately, the corresponding solutions with $\vec{\theta}_{\text {opt }}^{i}$ might better approximate the data from experiment $i$ than those with $\vec{\theta}_{\text {opt }}$ obtained using (42).

For the data from the first experiment we set

$$
\vec{\theta}_{0}^{1}=\left[\begin{array}{c}
1 \times 10^{-5} \\
8 \times 10^{-4} \\
2 \times 10^{-3} \\
4
\end{array}\right]
$$

and after optimization obtained

$$
\vec{\theta}_{\text {opt }}^{1}=\left[\begin{array}{c}
3.6712 \times 10^{-8} \pm 1.3978 \times 10^{-27} \\
7.3624 \times 10^{-4} \pm 5.8073 \times 10^{-25} \\
3.3313 \times 10^{-3} \\
4.4014 \times 10^{1} \pm 3.5449 \times 10^{-24}
\end{array}\right]
$$


With the second data set, we used $\vec{\theta}_{0}^{2}=\vec{\theta}_{0}^{1}$ and obtained

$$
\vec{\theta}_{\text {opt }}^{2}=\left[\begin{array}{c}
3.6955 \times 10^{-4} \pm 1.6159 \times 10^{-24} \\
4.0242 \times 10^{-3} \pm 8.4251 \times 10^{-25} \\
4.6266 \times 10^{-4} \\
1.9261 \times 10^{2} \pm 2.7588 \times 10^{-24}
\end{array}\right]
$$

A comparison of corresponding stresses with $\vec{\theta}_{\text {opt }}^{i}$ with the data for each experiment is presented in Figure 5. It is obvious from the second experiment that optimizing with its data set separately produces parameters that yield a model that more closely agrees with the data; the results from the first data set reflect the model's improved ability to achieve the data's peak, but in doing so a portion of the tail of the data is missed.

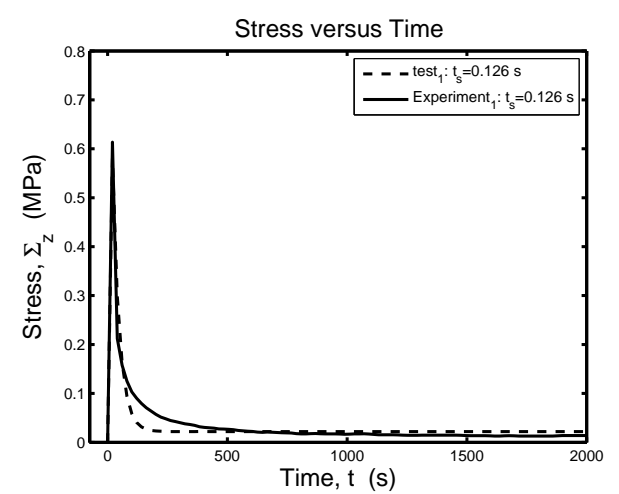

Cartilage Experiment 1

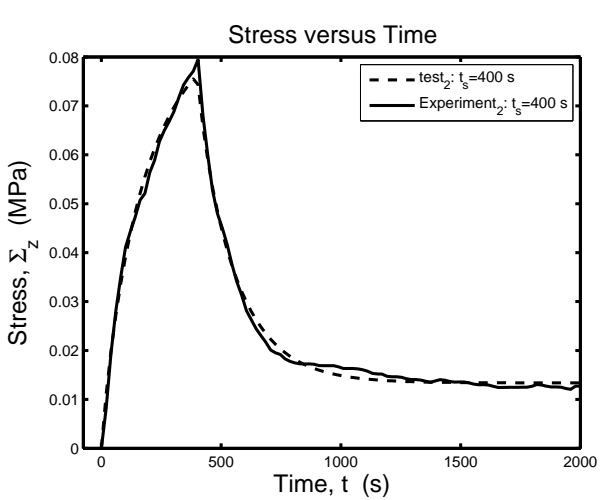

Cartilage Experiment 2

Figure 5: Stress calculations and experimental data for cartilage with ramp strains using separate cost functions (45).

\subsubsection{Stress versus strain model simulations}

Having estimated parameters for the stress-strain model (39), one can then use this model in simulations with various input strains to investigate the possible presence of features such as hysteresis. For example, when a stress-strain relation appears to possess a simple one-to-one graph in response to periodically oscillatory strain inputs, this indicates that there is little strain rate dependence in the system, i.e., no hysteresis is present. However, one expects that the graph of the stress-strain relation will appear as loops in response to such inputs when the system contains hysteresis.

In a series of simulations, various strain functions were input to the stress function of (39), and the stress-strain relations for each were graphed. For each of these simulations in this subsection, the parameter values used were those given in Table 2; the strain input functions vs. time, the resulting stress vs. time and the stress vs. strain relation are plotted below. For each simulation, the input strain function is taken on the interval from $t=0$ seconds to $t_{f}=200$ seconds. 
For the first simulation a sinusoidal input strain function given by

$$
\epsilon_{1}(t)=\epsilon_{\max }\left(-\frac{1}{2} \cos \left(\frac{10 \pi}{t_{f}} t\right)+1\right)
$$

is used to test the response of the system to cyclical input. This particular sinusoid was designed so that it is never negative and it ranges between 0 and $\epsilon_{\max }=0.05$. It can be seen from the results presented in Figure 6 that the stress exhibits hysteresis.

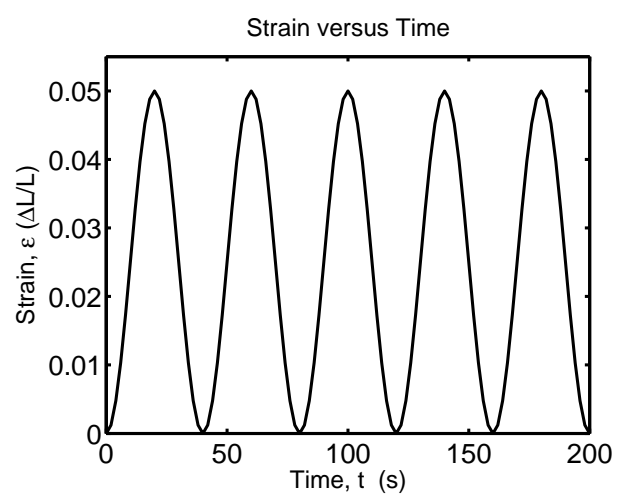

Strain vs. Time

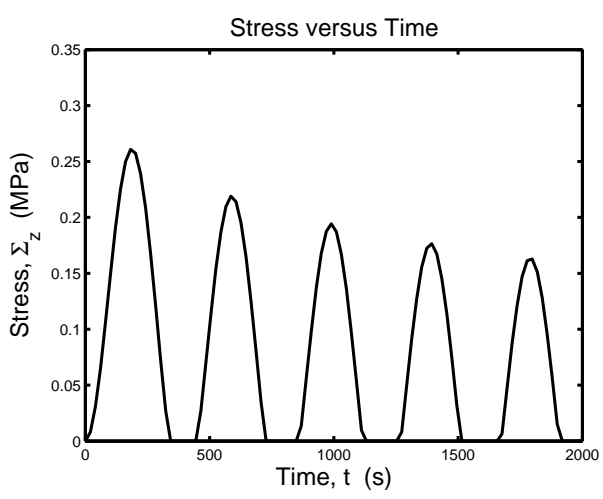

Stress vs. time

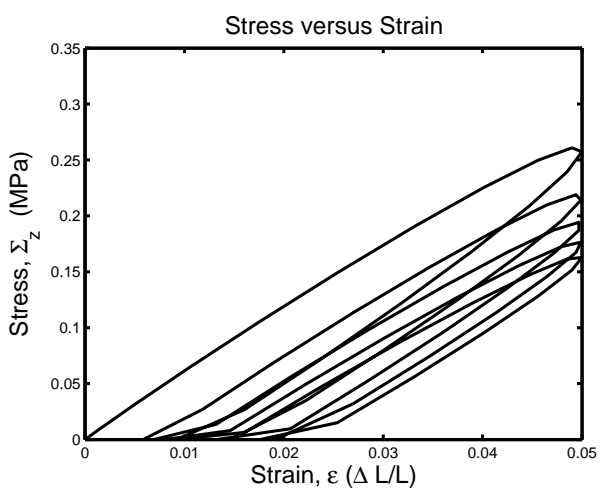

Stress vs. Strain

Figure 6: Graph of input strain $\epsilon_{1}(t)=\epsilon_{\max }\left(-\frac{1}{2} \cos \left(\frac{10 \pi}{t_{f}} t\right)+1\right)$, model stress vs. time, and model stress vs. strain relations for $\epsilon_{1}$ and $\Sigma_{z}$. 

by

In the next simulation we used sinusoidal strain function with increasing amplitude given

$$
\epsilon_{2}(t)=\epsilon_{\max }\left(-\frac{1}{2} \cos \left(\frac{10 \pi}{t_{f}} t\right)+1\right) \frac{t}{t_{f}} .
$$

This simulation was performed in an attempt to see if the stress of the system would vary in a way other than linearly with increasingly greater strain cycles. The results are depicted in Figure 7.

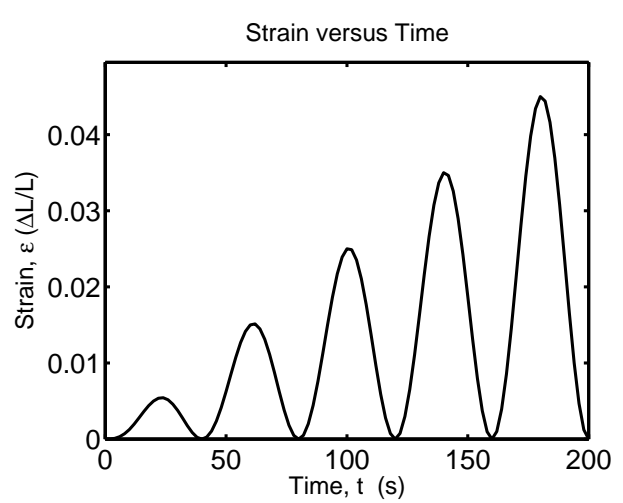

Strain vs. Time

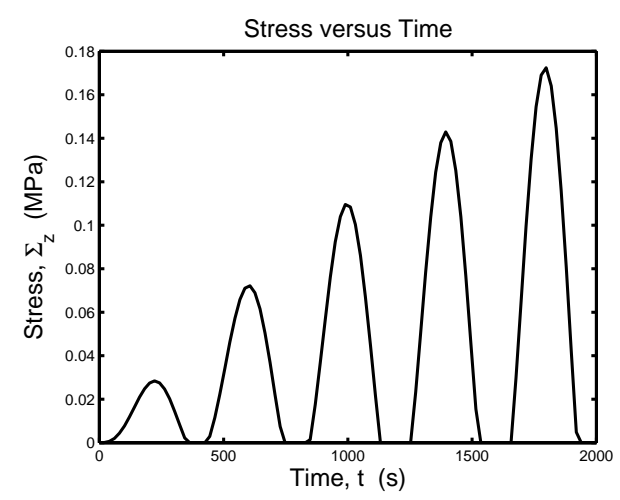

Stress vs. time

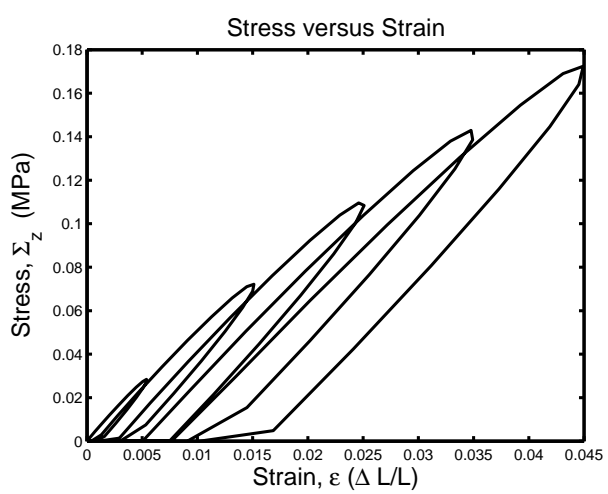

Stress vs. Strain

Figure 7: Graph of input strain $\epsilon_{2}(t)=\epsilon_{\max }\left(-\frac{1}{2} \cos \left(\frac{10 \pi}{t_{f}} t\right)+1\right) \frac{t}{t_{f}}$, model stress vs. time, and model stress vs. strain relations for $\epsilon_{2}$ and $\Sigma_{z}$.

For a third simulation, a simple bell curve for the strain input is employed. This particular strain function is chosen because of its simplicity. The function used to describe this strain is defined by

$$
\epsilon_{3}(t)=\frac{\epsilon_{\max }}{2}\left(-\cos \left(\frac{2 \pi}{t_{f}} t\right)+1\right)
$$

which is a single period of a cosine function. Results for this input strain function are presented in Figure 8, in which it may be seen that the stress-strain relation is a simple loop. 


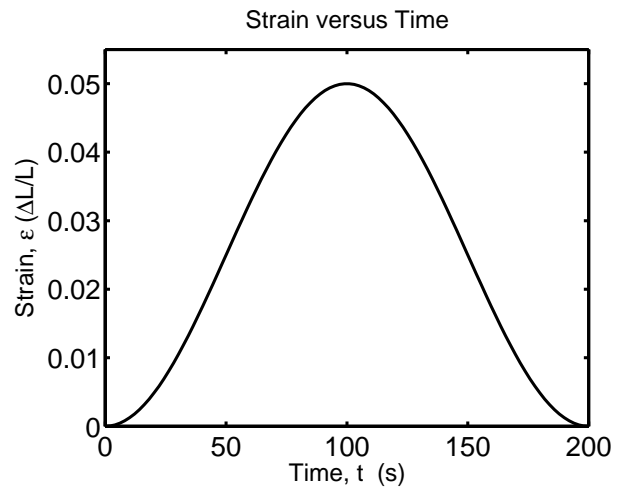

Strain vs. Time

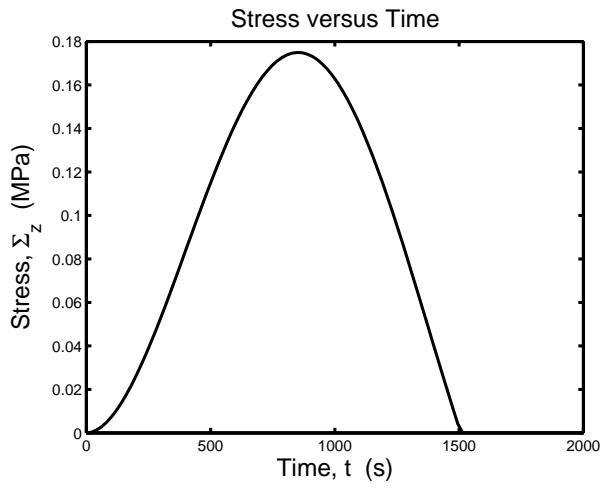

Stress vs. time

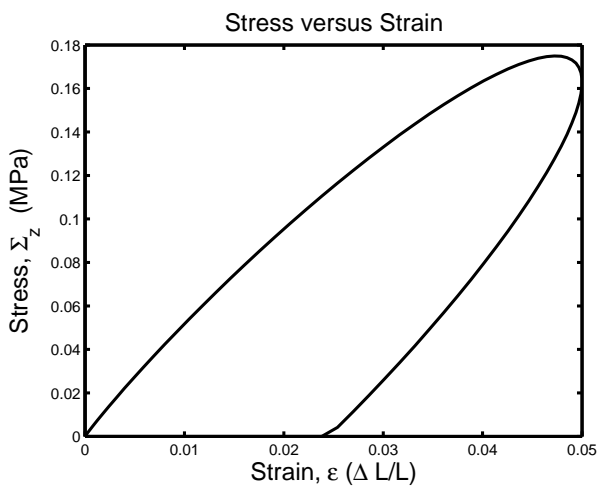

Stress vs. Strain

Figure 8: Graph of input strain $\epsilon_{3}(t)=\frac{\epsilon_{\max }}{2}\left(-\cos \left(\frac{2 \pi}{t_{f}} t\right)+1\right)$, model stress vs. time, and model stress vs. strain relations for $\epsilon_{3}$ and $\Sigma_{z}$. 


\subsection{Results for Polyisoprene}

We next investigated use of the model (39), or equivalently (43), with experimental data for polyisoprene. For the model calculations of the stress for polyisoprene, it is, of course, necessary to determine the particular parameters associated with that polymer (specifically the parameters $\tilde{a}, \tilde{b}, c, N$, and $\tilde{\mu}$ must be estimated).

We will choose the temperature to be at $298^{\circ} \mathrm{K}$ which is roughly room temperature. There have been many experiments conducted for polyisoprene at this temperature, and thus the amount of data from which we may derive first estimates of some of the parameters, especially the density parameter $c$ and the friction parameter $\zeta$ (a factor in both $\tilde{a}$ and $\tilde{b}$ ) which are affected by temperature, is substantial.

Using knowledge of the monomer chemical structure (see Figure 9) of polyisoprene, we can first calculate a parameter $x_{p}$ referred to as the degree of polymerization which is simply the number of monomers per single molecule. The degree of polymerization can be calculated using the equation

$$
x_{p}=\frac{M}{M_{0}},
$$

with molecular weight $M$ and the monomer weight $M_{0}$ (also referred to as the mass of the repeat unit). We can calculate the monomer weight using values observed in the monomer structure. We have that per monomer there are 5 carbon atoms and 8 hydrogen atoms.

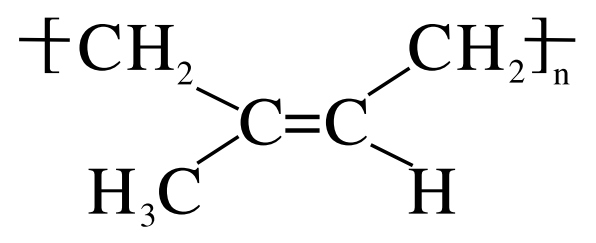

Figure 9: Chemical structure of polyisoprene polymer.

Thus, if we obtain the atomic weight for carbon (which is 12.01) and for hydrogen (which is 1.08) from any standard periodic table, we have that

$$
\begin{aligned}
M_{0} & =5(12.01)+8(1.008) \\
& =68.114 .
\end{aligned}
$$

For the molecular weight $M$, we use the value presented by Stille (Table 10.2, [36]) which is $M=350,000$. We are then able to calculate the degree of polymerization $x_{p}$ to be

$$
\begin{aligned}
x_{p} & =\frac{350,000}{68.114} \\
& \approx 5138,
\end{aligned}
$$

where we have rounded to the nearest whole monomer. The bond length $b$ is determined by segmentation of the polymer where the polymer chain is segmented into $N$ segments of 
length $b$. These are related to the contour length $L$ by $L=N b$. As argued in [23], $b$ can be approximated by $b \approx 8.415 \AA$ while $L=x_{p} b_{m}$ where $b_{m}$ is the average monomer length given approximately [37] by $4.602 \AA$ so that $L \approx 23647.25 \AA$. It then follows from $N=L / b$ that $N \approx 2810$.

We also need to determine an approximate value for the segment density $c$ of the sample. This is calculated using the formula (see equation (2) in [27]),

$$
c=\frac{\rho N_{A}}{M_{0}},
$$

where $\rho$ is the polymer density, $N_{A}$ is Avogadro's number, and $M_{0}$ is the monomer molecular weight. The value of $M_{0}$ was given above as $M_{0}=68.114$. For the polymer density we use the value given by Abdel-Goad, et al., [1], as $\rho=0.90 \times 10^{-24} \mathrm{~g} / \AA^{3}$ at $T=298 \mathrm{~K}$ (values range from $0.9 \times 10^{-24}$ to $1 \times 10^{-24} \mathrm{~g} / \AA^{3}$, [14],[16],[17],[18], but most commonly $0.9 \times 10^{-24}$ $\left.\mathrm{g} / \AA^{3}\right)$. The segment density is then

$$
\begin{aligned}
c & =\frac{\rho N_{A}}{M_{0}} \\
& =\frac{0.90 \times 10^{-24}\left(6.02 \times 10^{23}\right)}{68.114} \\
& \approx 0.007954 \text { segments } / \AA^{3} .
\end{aligned}
$$

We consider next the friction constant $\zeta$. In the Ferry text ([15]), the friction coefficient $\zeta$ is shown to be equal to the product of the number $x_{p}$ of monomers per molecule (the degree of polymerization), and the monomeric friction coefficient $\zeta_{m}$ (the friction provided by a single monomer). In Table 12-III on page 258 of [15], the monomer friction coefficient for polyisoprene (listed as unvulcanized Hevea rubber) at room temperature, $T=298^{\circ} \mathrm{K}$, is given in $\log$ form to be

$$
\log \left(\zeta_{m}\right)=-6.74 \text { dynes } \mathrm{s} / \mathrm{cm} .
$$

When converted to our units we obtain

$$
\zeta_{m}=1.18265 \times 10^{-6} \mathrm{~kg} / \mathrm{s} .
$$

Then when we apply the formula for $\zeta$ we find that

$$
\begin{aligned}
\zeta & =5138 \zeta_{m} \\
& \approx 0.006076 \mathrm{~kg} / \mathrm{s}
\end{aligned}
$$

is the friction present in a single strand of the polymer.

All that remains is the step-length $a$. Doi and Edwards [10] define $a$ in their equation (6.4) by

$$
L=\frac{N b^{2}}{a},
$$


where $L$ is the contour length (which was calculated earlier to be approximately 23647.25 $\AA$ ) and $N$ is the number of segments of length $b$. (We note that $N b^{2}$ is the mean square end-to-end distance $\left\langle r^{2}\right\rangle_{0}$ of the chain [10]). We thus find $a=8.415 \AA$. We can then use these values of $a, b$ and $\zeta$ to provide initial estimates for $\tilde{a}$ and $\tilde{b}$. We collect these and other pertinent parameters used for the polyisoprene estimation procedures in Table 3.

Table 3: Fixed parameters along with initial values used for polyisoprene stress versus strain estimation.

\begin{tabular}{|l|c|l|}
\hline Parameter & Abbreviation & Value \\
\hline Temperature & $T$ & $298^{\circ} \mathrm{K}$ \\
Segments/Chain & $N$ & 2810 \\
Boltzmann's Constant & $k_{B}$ & $1.3806505 \times 10^{-23} \mathrm{~J} / \mathrm{K}$ \\
Disengagement constant & $\tilde{a}_{0}$ & $4.3026 \times 10^{-1} \AA^{2} \mathrm{~kg} / \mathrm{s}$ \\
Rouse constant & $\tilde{b}_{0}$ & $4.3026 \times 10^{-1} \AA^{2} \mathrm{~kg} / \mathrm{s}$ \\
Segments/Volume & $c_{0}$ & $7.9540 \times 10^{-3} 1 / \AA^{3}$ \\
Young's constant & $\tilde{\mu}_{0}$ & $1.2572 \times 10^{-5} \mathrm{MPa} \AA^{3}$ \\
\hline
\end{tabular}

\subsubsection{Polyisoprene parameter estimation results}

Having determined a set of approximate parameters related to natural rubber (polyisoprene), we then used these as an initial guess in an OLS estimation procedure for the "best" parameters in (39). The data used in the OLS was provided by a graph from the text by Riande, et al., [33]. We used this data to define an input strain function in the form of a simple hat function

$$
\epsilon(t)=\left\{\begin{array}{ll}
\frac{4}{t_{f}} t & t \leq \frac{t_{f}}{2} \\
-\frac{4}{t_{f}}\left(t-t_{f}\right) & t>\frac{t_{f}}{2}
\end{array},\right.
$$

as graphed in Figure 10, with a maximum value of $\epsilon_{\max }=2$ and $t_{f}=500$ seconds. We used

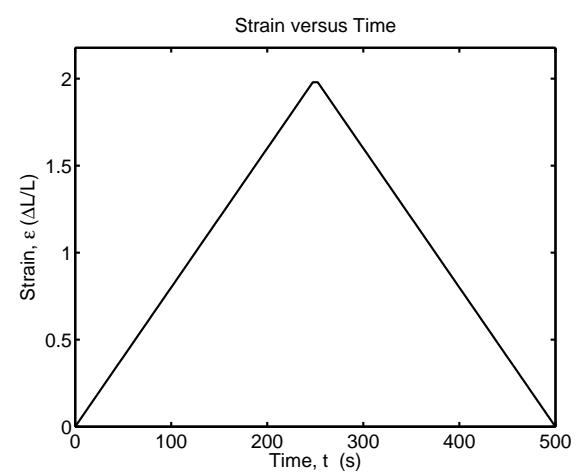

Figure 10: Strain function from which the stress for the polyisoprene simulations are carried out. 
this hat function as input for stick-slip model (39) and computed the corresponding stress to use as the model in an OLS cost functional

$$
C(\vec{\theta})=\sum_{j=1}^{100}\left|\Sigma_{z}\left(t_{j} ; \vec{\theta}\right)-y^{j}\right|^{2},
$$

where the stress data points obtained from the graph from [33] are referred to as $\left\{y^{j}\right\}_{j=1}^{100}$, with each $y^{j}$ representing the stress for $\epsilon\left(t_{j}\right)$ for $t_{j}$ the uniformly spaced time points on the interval from $t=0$ to $t=500$ seconds. This stress function $\Sigma_{z}\left(t_{j} ; \vec{\theta}\right)=\Sigma_{z}\left(t_{j} ; \vec{\theta}, i\right)$ of $(43)$ and the stress data was used to estimate the parameters in the model. In (43) we used $\lambda_{i}(t)=\lambda(t)=1+\epsilon(t)$ for $\epsilon$ given in (49), and we again choose the limits of summation to be $M=10$ and $M^{\prime}=21$. We carried out estimation of the parameters using the initial values for $\tilde{a}, \tilde{b}, c$ and $\tilde{\mu}$ given in Table 3 . The optimal values (with standard errors) found are

$$
\vec{\theta}_{\text {opt }}=\left[\begin{array}{c}
2.9746 \times 10^{-1} \pm 4.2663 \times 10^{-17} \\
5.7572 \times 10^{-1} \pm 1.6687 \times 10^{-18} \\
5.2495 \times 10^{-5} \\
2.2017 \times 10^{-2} \pm 1.0104 \times 10^{-18}
\end{array}\right]
$$

A graph of the corresponding model stress-strain curve using the optimal parameter values is compared to the experimental data in Figure 11. We note that while the basic scale

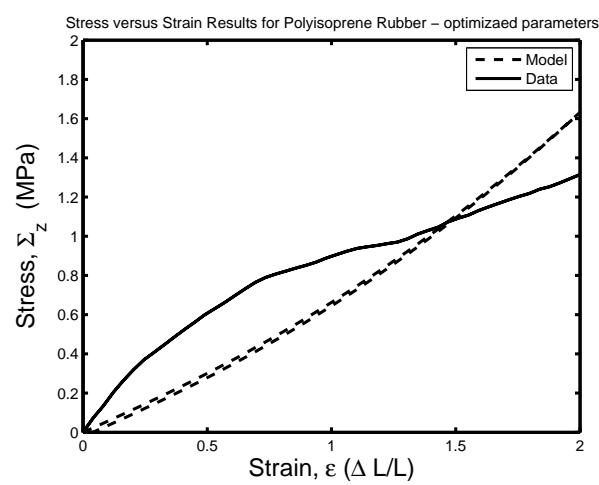

Stick-Slip/Rouse Hybrid Model and Comparative Data from [33]

Figure 11: Model stress versus strain simulation using optimal parameters compared to data for polyisoprene.

and trends of the two graphs are qualitatively similar, it is interesting to note that there is little to no hysteresis exhibited by pure natural rubber, both in our simulations and in the comparative data. Moreover, there are nonlinear aspects of the data that clearly are not captured by the model. We recall the linearization assumption of (15) which might suggest difficulties for the model when used with nonlinear materials. 


\subsection{Polyisoprene with carbon black reinforcement}

Natural rubber (polyisoprene), as seen in the graph of Figure 11, exhibits mild nonlinear behavior but very little hysteresis. However, most rubber-based products contain rubber composites (or filled rubber) and it has been known for a long time that various properties of the composite are affected by incorporating substances (a common practice for industrial products) such as carbon black or colloidal carbon into the polyisoprene (i.e., by vulcanizing it). In the paper by Parkinson [32], it is noted that carbon black particles are typically spherical and range in diameter from roughly 50 to $5000 \AA$, although other sources have it as being between 10 to $10000 \AA$. When introduced into the raw polymer, the polymer strands attach to these spheres, restricting the flow of the polymers.

One side effect of the addition of carbon black to polyisoprene is the production of significant hysteresis in the stress-strain relation of the material. In addition, other, more desirable, effects including an increase in the stiffness of the material, resistance to absorption of other fluids, abrasion resistance and heat resistance in the composite may be produced. These effects can often be tailored to the desired levels by varying the amount and type of carbon black introduced to the raw polymer. For example, carbon black reinforced rubbers are used in both car tires and in rubber stoppers. At first glance, these items may appear to be made of different material, because tire rubber is so much stiffer than a typical rubber stopper; however they only differ in their carbon black content.

In the text by Riande, et al., [33], there is an experimental data set for rubber reinforced with carbon black (graphed here as the solid curve in Figure 13 below) undergoing deformations. While the concentration of the carbon black within the sample used in the experiment presented in that graph is not known, it is possible, if we use the same inverse problem methods as those of subsections 6.1.1, to estimate the parameters in our model (43).

The data was first extracted from the graph of [33], in a manner similar to that used in section 6.1.1. It was assumed that the experiment takes place on the interval $0 \leq t \leq 500$ and that the strain function (which is not known to us) was approximately piecewise linear. Under this assumption an approximate strain function, obtained from the data set, was found by determining the lines which contain the maximum and minimum points of the strain and defining the strain piecewise. The strain function is then of the form

$$
\epsilon_{R C B}(t)= \begin{cases}(2.07051 / 165) t & 0 \leq t \leq 170 \\ -(1.9257 / 165)(t-330)+0.1448 & 170<t<330 \\ (1.98671 / 170)(t-330)+0.1448 & t \geq 330\end{cases}
$$

The graph of this strain function is given in Figure 12.

The stress data points obtained from the graph from [33] are referred to as $\left\{y^{j}\right\}_{j=1}^{100}$, where each $y^{j}$ is represents the stress for $\epsilon_{R C B}\left(t_{j}\right)$ for $t_{j}$ the uniformly spaced time points on the interval $[0,500]$. This strain function and the stress data were used to estimate the parameters in the model (43). We assume that we are working with approximately the same number of segments as from Section 6.2.1 and take $N=2000$; we use the same temperature, $T=298^{\circ} \mathrm{K}$. 


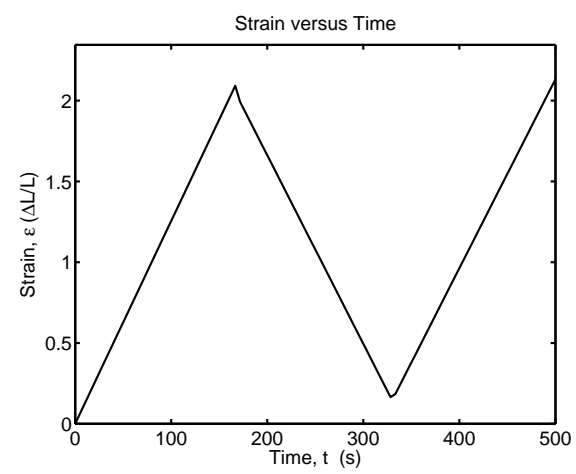

Figure 12: Strain function from which the stress for the polyisoprene with carbon black reinforcement simulations are carried out.

As we did earlier, we need to find the parameters $\tilde{a}, \tilde{b}, c$, and $\tilde{\mu}$. We again define the vector $\vec{\theta}$ of parameters to be

$$
\vec{\theta}=\left[\begin{array}{c}
\tilde{a} \\
\tilde{b} \\
c \\
\tilde{\mu}
\end{array}\right]
$$

The function $\lambda$ is given by $\lambda(t)=1+\epsilon_{R C B}(t)$, where $\epsilon_{R C B}(t)$ is defined as above, with a corresponding piecewise constant derivative $\lambda^{\prime}$. The cost function used in the Nelder-Mead algorithm was the same as that given in (50).

If the initial guess is taken to be

$$
\vec{\theta}_{0}=\left[\begin{array}{c}
1.0 \times 10^{-3} \\
1.0 \times 10^{-3} \\
1.0 \times 10^{-1} \\
10
\end{array}\right]
$$

then the optimal value found is

$$
\vec{\theta}_{\text {opt }}=\left[\begin{array}{c}
1.5556 \times 10^{-4} \pm 4.9263 \times 10^{-24} \\
6.5350 \times 10^{-4} \pm 9.2709 \times 10^{-25} \\
3.4526 \times 10^{-4} \\
2.2248 \times 10^{2} \pm 1.1351 \times 10^{-22}
\end{array}\right] .
$$

The fixed and optimal parameter values used in the model simulation for the polyisoprene with carbon black reinforcement are collected in Table 4. The model simulations are compared to the experimental data from [33] in Figure 13.

As can be seen from the graph, the model does exhibit hysteresis in the polyisoprene simulations (as evidenced by the presence of loops in the stress-strain curve). However, the model does not capture very well the nonlinearities in the data. We recall that our 
Table 4: Optimal parameters used for polyisoprene with carbon black reinforcement stress versus strain simulation.

\begin{tabular}{|l|c|l|}
\hline Parameter & Abbreviation & Value \\
\hline Temperature & $T$ & $298^{\circ} \mathrm{K}$ \\
Segments/Chain & $N$ & 2000 \\
Boltzmann's Constant & $k_{B}$ & $1.3806505 \times 10^{-23} \mathrm{~J} / \mathrm{K}$ \\
Disengagement constant & $\tilde{a}_{\text {opt }}$ & $1.5556 \times 10^{-4} \AA^{2} \mathrm{~kg} / \mathrm{s}$ \\
Rouse constant & $\tilde{b}_{\text {opt }}$ & $6.5350 \times 10^{-4} \AA^{2} \mathrm{~kg} / \mathrm{s}$ \\
Segments/Volume & $c_{\text {opt }}$ & $3.4526 \times 10^{-4} 1 / \AA^{3}$ \\
Young's constant & $\tilde{\mu}_{\text {opt }}$ & $2.2248 \times 10^{2} \mathrm{MPa} \AA^{3}$ \\
\hline
\end{tabular}

model is based on the linear Rouse model (4) (which is linear due to the assumptions on the underlying potentials) and the linearization assumption (15). Moreover, both (24) and (28) are assumptions that are linear in nature (as opposed to the nonlinear Johnson-Stacer type ratio assumptions of $[6,7]$ which led to full nonlinear models for hysteretic constitutive laws there). To provide a better fit to the carbon-black filled polyisoprene data, one may require nonlinear assumptions in the model development similar to those in $[6,7]$, or the more appropriate assumption in (2) of a Lennard-Jones type higher order potential [28, 30] which permits repulsive forces as "beads" in the molecular chain become close. On a more positive note, it is clear that it is possible to portray the qualitative trends in hysteretic and non-hysteretic materials with the present model.

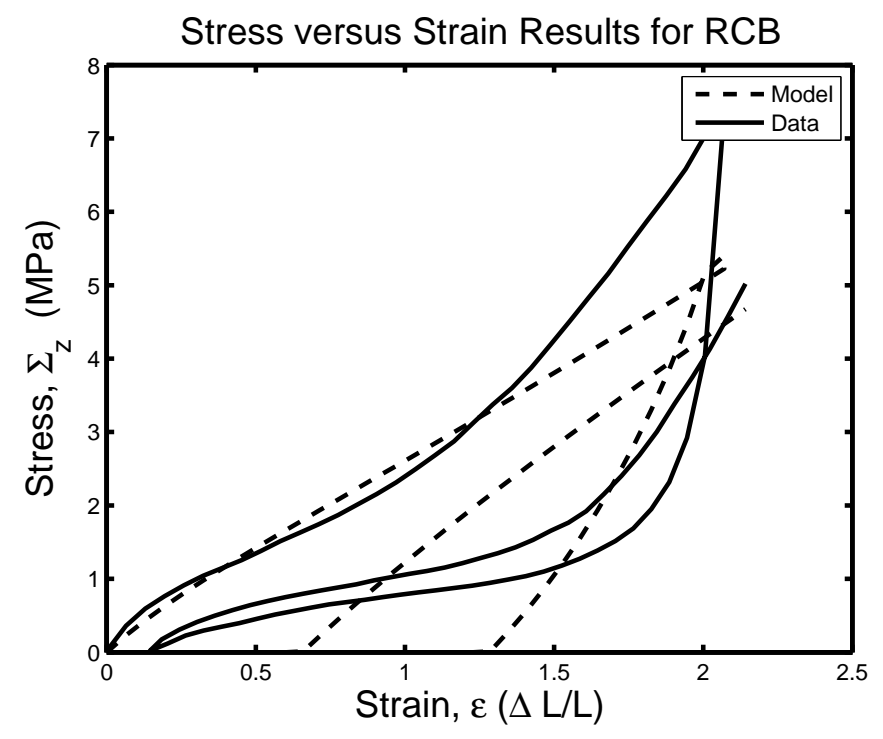

Stick-Slip/Rouse Hybrid Model

Figure 13: Stress versus strain calculation and comparative data for polyisoprene with carbon black reinforcement simulation. 


\section{Conclusions}

In this paper we have derived a new molecular based model to describe hysteresis in polymers and polymer-like materials. The models, based on a linear stick-slip assumption, while capturing some of the hysteresis in several types of materials, do not capture especially well the shapes of the nonlinearities in the hysteresis loops. While these models are a useful initial effort, further development along the lines of the nonlinear stick-slip assumptions of $[6,7]$ is needed.

\section{Appendix}

In this section Einstein notation (summation over repeated indices) will be used, so the expression $\sum_{\mu} E_{\alpha \mu}\left(t_{i}\right) E_{\beta \mu}\left(t_{i}\right)$ (our previous notation) is the same as $E_{\alpha \mu}\left(t_{i}\right) E_{\beta \mu}\left(t_{i}\right)$.

We have claimed that the quantity

$$
\left\langle X_{p}^{\alpha}\left(t_{i}+\Delta t\right) X_{p}^{\beta}\left(t_{i}+\Delta t\right)\right\rangle-\left\langle X_{p}^{\alpha}\left(t_{i}^{-}\right) X_{p}^{\beta}\left(t_{i}^{-}\right)\right\rangle
$$

can be approximated by

$$
\left.\frac{d}{d t}\left[B_{\alpha \beta}(\mathbf{E}(t))\right]\right|_{t=t_{i}} \Delta t
$$

To verify this claim, we will consider two cases: the configuration gradient $E_{\alpha \mu}\left(t_{i}\right)$ is discontinuous (case 1) and the configuration gradient $E_{\alpha \mu}\left(t_{i}\right)$ is continuous (case 2).

Let $E_{\alpha \mu}\left(t_{i}\right)=\delta_{\alpha \mu}+\Delta_{\alpha \mu}\left(t_{i}^{+}\right)$and let $\delta_{\alpha \mu}$ be the current configuration of the polymer. Case 1 can be resolved by observing that

$$
\left\langle X_{p}^{\alpha}\left(t_{i}+\Delta t\right) X_{p}^{\beta}\left(t_{i}+\Delta t\right)\right\rangle-\left\langle X_{p}^{\alpha}\left(t_{i}^{-}\right) X_{p}^{\beta}\left(t_{i}^{-}\right)\right\rangle
$$

can be written, using arguments based on those presented in Section 3 and grouping higher order terms (H.O.T.), as

$$
\begin{aligned}
= & C\left\{\Delta_{\beta \alpha}\left(t_{i}^{+}\right)+\Delta_{\alpha \beta}\left(t_{i}^{+}\right)\right\}+\text {H.O.T. } \\
= & C \frac{1}{\Delta t}\left\{\left[\delta_{\alpha \mu}+H(\Delta t) \Delta_{\alpha \mu}\left(t_{i}^{+}\right)\right]\left[\delta_{\beta \mu}+H(\Delta t) \Delta_{\beta \mu}\left(t_{i}^{+}\right)\right]\right. \\
& \quad-\left[\delta_{\alpha \mu}+H(0) \Delta_{\alpha \mu}\left(t_{i}^{+}\right)\right]\left[\delta_{\beta \mu}+H(0) \Delta_{\beta \mu}\left(t_{i}^{+}\right)\right\} \Delta t+\text { H.O.T. } \\
& =C \frac{1}{\Delta t}\left\{E_{\alpha \mu}\left(t_{i}+\Delta t\right) E_{\beta \mu}\left(t_{i}+\Delta t\right)-E_{\alpha \mu}\left(t_{i}\right) E_{\beta \mu}\left(t_{i}\right)\right\} \Delta t+\text { H.O.T. } \\
\approx & C \frac{1}{\Delta t}\left\{E_{\alpha \mu}\left(t_{i}+\Delta t\right) E_{\beta \mu}\left(t_{i}+\Delta t\right)-E_{\alpha \mu}\left(t_{i}\right) E_{\beta \mu}\left(t_{i}\right)\right\} \Delta t
\end{aligned}
$$

where $C$ is a constant and the function $H(x)$ is the Heaviside function $H(x)=0$ for $x \leq 0$ and $H(x)=1$ for $x>0$. Observe in (52) that

$$
\begin{aligned}
\lim _{\Delta t \rightarrow 0^{+}} \frac{\left\{E_{\alpha \mu}\left(t_{i}+\Delta t\right) E_{\beta \mu}\left(t_{i}+\Delta t\right)-E_{\alpha \mu}\left(t_{i}\right) E_{\beta \mu}\left(t_{i}\right)\right\}}{\Delta t} & =\lim _{\Delta t \rightarrow 0^{+}} \frac{\left(B_{\alpha \beta}\left(\mathbf{E}\left(t_{i}+\Delta t\right)\right)-B_{\alpha \beta}\left(\mathbf{E}\left(t_{i}\right)\right)\right)}{\Delta t} \\
& =\left.\frac{d}{d t}\left(B_{\alpha \beta}(\mathbf{E}(t))\right)\right|_{t=t_{i}} .
\end{aligned}
$$


This implies

$$
\left\langle X_{p}^{\alpha}\left(t_{i}^{+}\right) X_{p}^{\beta}\left(t_{i}^{+}\right)\right\rangle-\left.\left\langle X_{p}^{\alpha}\left(t_{i}^{-}\right) X_{p}^{\beta}\left(t_{i}^{-}\right)\right\rangle \approx C \frac{d}{d t} B_{\alpha \beta}(\mathbf{E}(t))\right|_{t=t_{i}} \Delta t
$$

For case 2 we will suppose $E_{\alpha \mu}(t)$ is differentiable from the right at $t=t_{i}$. Thus $E_{\alpha \mu}^{\prime}\left(t_{i}^{+}\right)$ exists, $E_{\alpha \mu}\left(t_{i}^{+}\right)=\lim _{\Delta t \rightarrow 0^{+}} E_{\alpha \mu}\left(t_{i}+\Delta t\right)$ and $E_{\alpha \mu}\left(t_{i}+\Delta t\right) \approx \delta_{\alpha \mu}+E_{\alpha \mu}^{\prime}\left(t_{i}\right) \Delta t\left(\delta_{\alpha \mu}\right.$ denotes the configuration before the deformation). Again, if arguments based on those presented in Section 3 are used, then

$$
\left\langle X_{p}^{\alpha}\left(t_{i}+\Delta t\right) X_{p}^{\beta}\left(t_{i}+\Delta t\right)\right\rangle-\left\langle X_{p}^{\alpha}\left(t_{i}^{-}\right) X_{p}^{\beta}\left(t_{i}^{-}\right)\right\rangle
$$

can be approximated by

$$
\begin{aligned}
C\left(E_{\alpha \beta}^{\prime}\left(t_{i}\right)+E_{\beta \alpha}^{\prime}\left(t_{i}\right)\right) \Delta t+\text { H.O.T. } & =C\left(E_{\alpha \mu}^{\prime}\left(t_{i}\right) E_{\beta \mu}\left(t_{i}\right)+E_{\beta \nu}^{\prime}\left(t_{i}\right) E_{\alpha \nu}\left(t_{i}\right)\right) \Delta t+\text { H.O.T. } \\
& =\left.C \frac{d}{d t}\left(E_{\alpha \mu} E_{\beta \mu}\right)\right|_{t=t_{i}} \Delta t+\text { H.O.T. } \\
& \left.\approx C \frac{d}{d t} B_{\alpha \beta}(\mathbf{E}(t))\right|_{t=t_{i}} \Delta t .
\end{aligned}
$$

The above arguments justify the approximation

$$
\left\langle X_{p}^{\alpha}\left(t_{i}+\Delta t\right) X_{p}^{\beta}\left(t_{i}+\Delta t\right)\right\rangle-\left.\left\langle X_{p}^{\alpha}\left(t_{i}^{-}\right) X_{p}^{\beta}\left(t_{i}^{-}\right)\right\rangle \approx C \frac{d}{d t} B_{\alpha \beta}(\mathbf{E}(t))\right|_{t=t_{i}} \Delta t
$$

in both the continuous and discontinuous cases.

\section{Acknowledgements}

This research was supported in part by the US Air Force Office of Scientific Research under grant AFOSR-FA9550-04-1-0220. It was also supported in part by a GAANN Fellowship to JRS under grant U.S. Department of Education P200A030277.

\section{References}

[1] M. Abdel-Goad, W. Pyckhout-Hintzen, S. Kahle, J. Allgaier, D. Richter and L.J. Fetters, Rheological properties of 1,4-polyisoprene over a large molecular weight range, Macromolecules, 37 (2004), 8135-8144.

[2] H.T. Banks, J.B. Hood and N.G. Medhin, A molecular based model for polymer viscoelasticity: Intra- and inter-molecular variability. Technical Report CRSC-TR04-39, NCSU, December, 2004; Applied Mathematical Modelling, submitted.

[3] H.T. Banks, G.M. Kepler, H.K. Nguyen, and J. Webster-Cyriaque, Inverse problems and model validation: An example from latent virus reactivation, Technical Report CRSC-TR06-18, NCSU, August, 2006; J. Inverse and Ill-posed Problems, submitted. 
[4] H.T. Banks and N.J. Lybeck, Modeling methodology for elastomer dynamics, Technical Report CRSC-TR96-29, NCSU, September, 1996; Systems and Control in the TwentyFirst Century, (C. Byrnes, et al, eds.), Birkhäuser, PSCT22, 1996, pp. 37-50.

[5] H.T. Banks, N.J. Lybeck, M.J. Gaitens, B.C. Muñoz, and L.C. Yanyo, Modeling the dynamic mechanical behavior of elastomers, Technical Report CRSC-TR96-26, NCSU, September, 1996.

[6] H.T. Banks, N.G. Medhin and G.A. Pinter, Multiscale considerations in modeling of nonlinear elastomers, Technical Report CRSC-TR03-42, NCSU, October, 1996; International Journal of Computational Methods in Engineering Science and Mechanics, to appear.

[7] H.T. Banks, N.G. Medhin and G.A. Pinter, Nonlinear reptation in molecular based hysteresis models for polymers, Technical Report CRSC-TR03-45, NCSU, December, 1996; Quarterly of Applied Mathematics, to appear.

[8] H.T. Banks, G.A. Pinter, L.K. Potter, M.J. Gaitens and L.C. Yanyo, Modeling of quasistatic and dynamic load responses of filled viscoelastic materials, Technical Report CRSC-TR98-48, NCSU, December, 1998; Chapter 11 in Mathematical Modeling: Case Studies from Industry (E. Cumberbatch and A. Fitt, eds.), Cambridge University Press, (2001), pp. 229-252.

[9] H.T. Banks, G.A. Pinter, L.K. Potter, B.C. Muñoz, and L.C. Yanyo, Estimation and control related issues in smart material structures and fluids, Technical Report CRSCTR98-02, NCSU, January, 1998; Optimization Techniques and Applications (L. Caccetta, et al., eds.), Curtain Univ. Press, July 1998, pp. 19-34.

[10] M. Doi and S.F. Edwards, The Theory of Polymer Dynamics, Oxford University Press, New York, 1986.

[11] M. Doi, Introduction to Polymer Physics, Clarendon Press, Oxford, 1996.

[12] Jiro Doke, Grabit.m, The MathWorks MatLab Central Website, March 17, 2005, http://www.mathworks.com/matlabcentral/fileexchange (accessed April 13, 2005).

[13] M. Davidian and D.M. Giltinan, Nonlinear Models for Repeated Measurement Data, Chapman and Hall, London, 1995.

[14] M. Doxastakis, D.N. Theodorou, G. Fytas, F. Kremer, R. Faller, F. Müller-Plathe and N. Hadjichristidis, Chain and local dynamics of polyisoprene as probed by experiments and computer simulations, Journal of Chemical Physics, 119 (203), 6883-6894.

[15] J.D. Ferry, Viscoelastic Properties of Polymers, John Wiley and Sons, Inc., New York, 1961. 
[16] L.J. Fetters, D.J. Lohse and W.W. Graessley, Chain dimensions and entanglement spacings in dense macromolecular systems, Journal of Polymer Science: Part B: Polymer Physics, 37 (1999), 1023-1033.

[17] L.J. Fetters, D.J. Lohse and S.T. Molner, Packing length influence in linear polymer melts on the entanglement, critical, and reptation molecular weights, Macromolecules, 32 (1999), 6847-6851.

[18] L.J. Fetters, D.J. Lohse, D. Richter, T.A. Witten and A. Zirkel, Connection between polymer molecular weight, density, chain dimensions, and melt viscoelastic properties, Macromolecules, 27 (1999), 4639-4647.

[19] Y.C. Fung, Foundations of Solid Mechanics, Prentice-Hall, Englewood Cliffs, NJ, 1965.

[20] Y.C. Fung, Biomechanics : Mechanical Properties of Living Tissues, Springer-Verlag, New York, 1993.

[21] D.P. Fyhrie and J.R. Barone, Polymer dynamics as a mechanistic model for the flowindependent viscoelasticity of cartilage, J. Biomech. Eng., 125 (2003), 578-584.

[22] A.R. Gallant, Nonlinear Statistical Models, John Wiley \& Sons, Inc., New York, 1987.

[23] J.B. Hood, Molecular-based Models for Viscoelasticity of Polymers, Ph.D. Thesis, North Carolina State University, Raleigh, NC, July, 2005

[24] C.Y. Huang, V.C. Mow and G.A. Ateshian, The role of flow-independent viscoelasticity in the biphasic tensile and compressive responses of articular cartilage, J. Biomech. Eng., 123 (2001), 410-417.

[25] R.I. Jennrich, Asymptotic properties of non-linear least squares estimators, Ann. Math. Statist., 40 (1969), 633-643.

[26] A.R. Johnson and R.G. Stacer, Rubber viscoelasticity using the physically constrained system's stretches as internal variables, Rubber Chemistry and Technology, 66 (1993), $567-577$.

[27] R.G. Larson, T. Sridhar, L.G. Leal, G.H. McKinley, A.E. Likhtman and T.C.B. McLeish, On the definitions of entanglement spacing and time constants in the tube model, Journal of Rheology, 47 (2003), 809-818.

[28] J.E. Lennard-Jones, Cohesion, Proc. Physical Soc.(London), 43 (1931), 461-482.

[29] J.E. Marsden and T.J.R. Hughes, Mathematical Foundations of Elasticity, PrenticeHall, Englewood Cliffs, NJ, 1983.

[30] D.A. McQuarrie, Statistical Thermodynamics, Harper \& Row, New York, 1973. 
[31] R.W. Ogden, Non-Linear Elastic Deformations, Ellis Horwood Limited, Chichester, 1984.

[32] D. Parkinson, The reinforcement of rubber by carbon black, British Journal of Applied Physics, 2 (1951), 273-280.

[33] E. Riande, R. Diaz-Calleja, M.G.Prolongo, R.M. Masegosa and C. Salom, Polymer Viscoelasticity - Stress and Strain in Practice, Marcel Dekker, Inc, New York, 2000.

[34] R.S. Rivlin, Large elastic deformations of isotropic materials, I, II, III., Phil. Trans. Roy. Soc. A 240 (1948), 459-525.

[35] G.A.F. Seber and C.J. Wild, Nonlinear Regression, John Wiley \& Sons, Inc., New York, 1989.

[36] J.K. Stille, Introduction to Polymer Chemistry, John Wiley and Sons, Inc., New York, 1962.

[37] A.F. Terzis, Bisdisperse melt polymer brush studied by self-consistent field model, Polymer, 43 (2002), 2435-2444.

[38] L.R.G. Treloar, The Physics of Rubber Elasticity, Clarendon, Oxford 1975.

[39] I.M. Ward, Mechanical Properties of Solid Polymers, J. Wiley \& Sons, New York, 1983. 